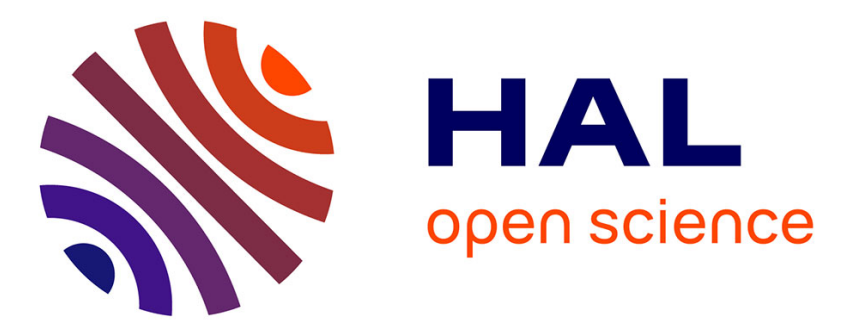

\title{
Geometrical defect identification of a SCARA robot from a vector modeling of kinematic joints invariants
}

Hélène Chanal, Jean Baptiste Guyon, Adrien Koessler, Quentin Dechambre, Benjamin Boudon, Benoît Blaysat, Nicolas Bouton

\section{- To cite this version:}

Hélène Chanal, Jean Baptiste Guyon, Adrien Koessler, Quentin Dechambre, Benjamin Boudon, et al.. Geometrical defect identification of a SCARA robot from a vector modeling of kinematic joints invariants. Mechanism and Machine Theory, 2021, 162, pp.104339. 10.1016/j.mechmachtheory.2021.104339 . hal-03182929

\author{
HAL Id: hal-03182929 \\ https://hal.uca.fr/hal-03182929
}

Submitted on 26 Mar 2021

HAL is a multi-disciplinary open access archive for the deposit and dissemination of scientific research documents, whether they are published or not. The documents may come from teaching and research institutions in France or abroad, or from public or private research centers.
L'archive ouverte pluridisciplinaire HAL, est destinée au dépôt et à la diffusion de documents scientifiques de niveau recherche, publiés ou non, émanant des établissements d'enseignement et de recherche français ou étrangers, des laboratoires publics ou privés. 


\author{
Geometrical defect identification of a SCARA robot from a vector modeling of \\ kinematic joints invariants \\ Hélène Chanal (a), Jean Baptiste Guyon ${ }^{(a)}$, Adrien Koessler ${ }^{(a)}$, Quentin \\ Dechambre $^{(a)}$, Benjamin Boudon ${ }^{(a)}$, Benoit Blaysat ${ }^{(a)}$, Nicolas Bouton ${ }^{(a)}$ \\ (a) Université Clermont Auvergne, CNRS, Clermont Auvergne INP, Institut Pascal, F- \\ 63000 Clermont-Ferrand, France. \\ * corresponding author: helene.chanal@sigma-clermont.fr
}

\begin{abstract}
:
This article introduces a new geometric vector modeling method of serial kinematic robot consistent with the identification process. This method is based on the definition of position and orientation of the robot joint invariants. For example, the invariant of the rotational joint is a straight-line (rotational joint axis). Thus, only independent geometrical parameters are introduced to model the joint axis position and orientation in space. Note that, the orientation is not constrained as in the Denavit-Hartenberg ( $\mathrm{DH})$ formalism. This article presents the methodology to define these geometrical parameters and the geometrical model. In this context, the identification method relies on "Circle Point Analysis". The points are measured with a laser tracker. Indeed, with a relevant processing of the measured points, we directly identify the invariants of joints. This method is applied to a SCARA robot geometric modeling. After an identification process, this methodology allows improving inverse kinematic error compared to the classical DH geometrical model with first and second-order defects. Moreover, the obtained residual error mean value is close to the accuracy of the measurement process.
\end{abstract}

Keywords: geometrical modeling, geometrical identification, SCARA robot, Circle Point Analysis, joint invariant

\title{
1 Introduction
}

Improving the accuracy and productivity of a task frequently requires the implementation of a more or less autonomous process. It is in this context that manipulating robots were introduced. In this work, we focus on robots intended for "Pick and Place" operations. For this type of task, according to ISO9283 (ISO 9283,1993), the required performance criteria are: pose accuracy and repeatability, orientation accuracy, stabilization time, and static stiffness.

With the increase of robotic application requirements, a manual learning method is not possible and programming must be realized offline in a simulation environment [1]. Therefore, the control of pose accuracy of the robot is essential. It involves proposing a geometric model with its associated identification process [2]. The final accuracy of the robot depends on the model choice and identification process [3][4]. In this article, we propose a new method to model the geometric behavior of serial robots with the aim of improving their final accuracy after a classical identification process.

The geometric model is the mathematical description of the geometric behavior of the robot. This model expresses the pose (position of a particular point and orientation) of the endeffector in the fixed base coordinates system of the robot regarding the value of the active joints $q_{i}$. Its expression is linked to robot geometric parameters, which have a significant influence on the end-effector pose, and on situation parameters (degrees of freedom of the robot joints). 
A compromise must be found between the number of parameters considered, the complexity of the obtained model and the robustness of the identification process [1][5].

There are two main approaches to formalize the geometric model of a robot [6]. The first approach is composed of methods that directly identify the geometric parameters like the leg lengths or the angles between joint axes. This approach includes, for example, the DenavitHartenberg (DH) formalism (Figure 1) [7] or the Traveling Coordinate Systems (TCS) formalism [8]. The main advantage of these methods lies in their simplicity of implementation. In the case of the $\mathrm{DH}$ convention, the positioning and orientation of two adjacent joints require the introduction of four parameters [9]. The main drawback is the nature of these introduced geometric parameters. The application of this method is difficult in the case of two adjacent joints with collinear or intersecting axes [6][9]. In this case, the choice of $\vec{x}_{i-1}$ is not unique while it influences the definition of $\vec{z}_{i}$ orientation [2]. This drawback may lead to severe numerical difficulties for consecutive axes which are nominally parallel during the identification process. Indeed, small variations in axis alignment easily produce large changes in the geometric parameters [10]. Moreover, DH formalism does not ensure to consider and then identify joint axis orientation defect on $\left(\vec{x}_{i-1}, \vec{z}_{i-1}\right)$ plane (Figure 1). To solve this problem, additional parameters are added to position and orient coordinates systems fixed to adjacent robot elements. Thus, Veitschegger adds a second-order error term to take into account the orientation defect between two consecutive parallel joints [11]. However, this solution can introduce redundant parameters, which affect the robustness of the identification [12][13].

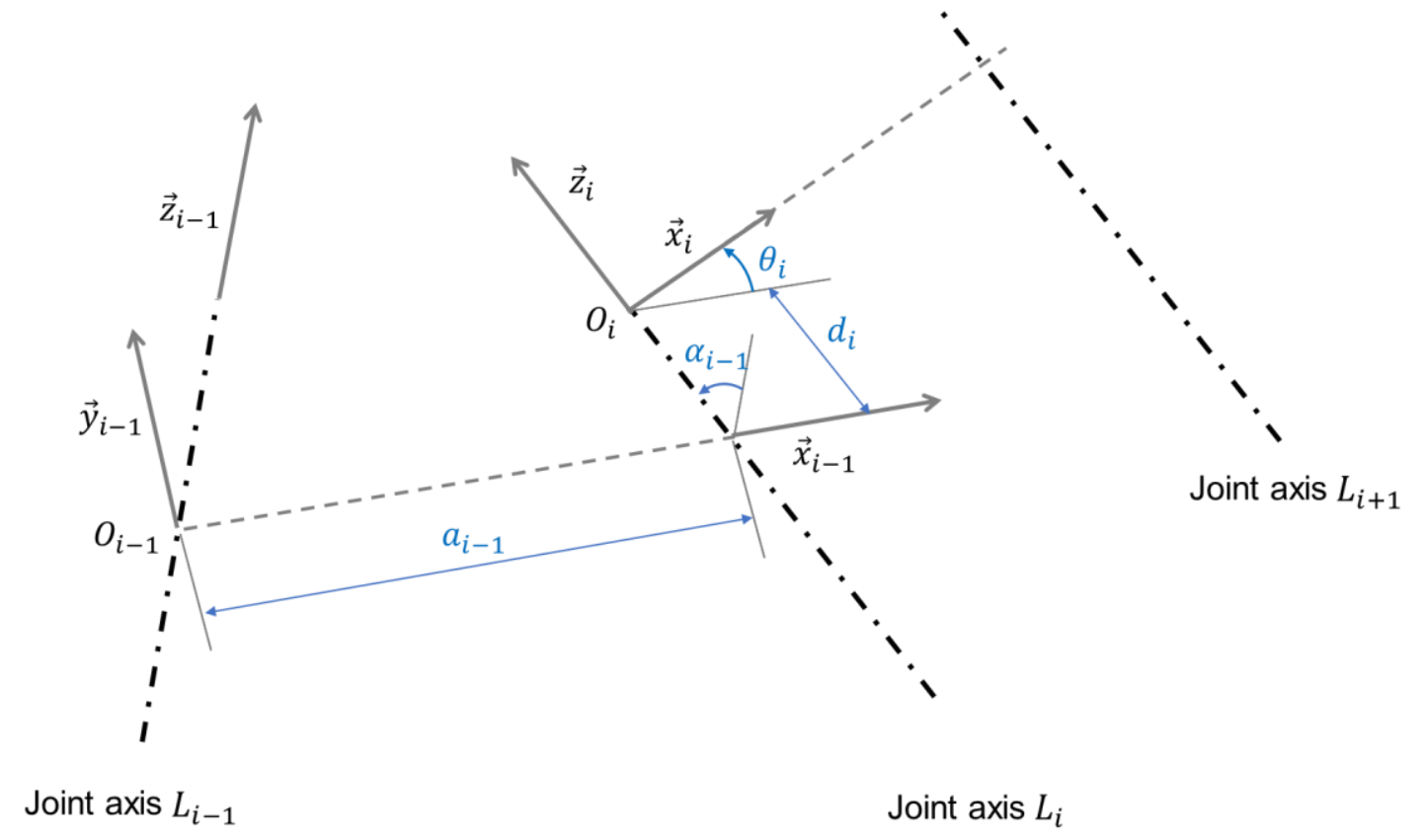

Figure 1: The DH geometric parameters in the case of a simple open structure [7]

The second approach concerns the methods used to identify the geometric parameters from the description of the kinematic movements of each joint. The geometric parameters are introduced during the definition of the reference model or "zero configuration" $\mathbf{g}_{\mathbf{s t}}(0)$ where all the active joint positions are null [1][14]:

$$
\mathbf{g}_{\mathbf{s t}}(0)=\left[\begin{array}{ll}
\mathbf{R} & \mathbf{p} \\
0 & 1
\end{array}\right]
$$

Where $\mathbf{R}$ is a $3 \times 3$ rotation matrix and $\mathbf{p}$ a $1 \times 3$ translation vector. 
From this reference model, the kinematic model $\mathbf{g}_{\mathbf{d}}$ is defined, for example, using "product-of-exponentials" (POE) [1][15][16] (Figure 2):

$$
\mathbf{g}_{\mathbf{d}}=e^{\widehat{\mathbf{s}_{\mathbf{1}}} q_{1}} \ldots e^{\widehat{\boldsymbol{s}_{\mathbf{n}}} q_{n}} \mathbf{g}_{\mathbf{s t}}(0)
$$

Where $\widehat{\mathbf{s}_{\mathbf{i}}}$ is the twist matrix associated with joint $i$ and $q_{i}$ is the joint position. The methodology to compute $e^{\widehat{s}_{\mathbf{i}} q_{i}}, i \in\{1, \ldots, n\}$ is explained in [1].

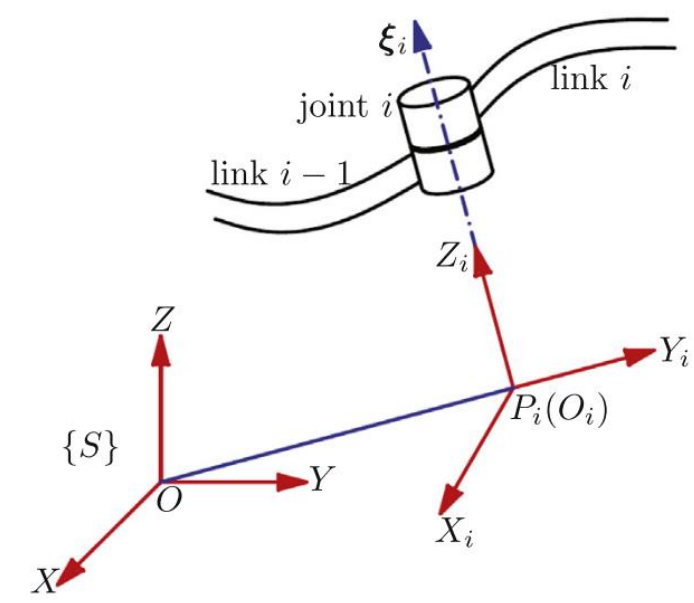

Figure 2: Link frame for a revolut joint for POE method [1]

The POE describes the configuration of a chain of bodies connected by lower pair joints. The kinematics is given in terms of joint screws [17].

In these methods, the definition of the reference model is not constrained by the coordinate system associated with each part. This leaves open the question of setting up redundant geometric parameters. By focusing on kinematic joint invariants, Yang developed a minimal kinematic model from a POE formulation [1]. Indeed, the number $C$ of independent geometric parameters necessary to describe the reference geometry of a serial robot is known according to [10]:

$$
C=4 R+2 P+6
$$

where $\mathrm{R}$ is the number of rotational joints and $\mathrm{P}$ is the number of prismatic joints. Indeed, according to Everett, 6 parameters are required to specify the position of the robot frame in the fixed coordinate system, 4 parameters are required to position and orient a rotation axis, and 2 parameters to orient a prismatic joint axis [10].

This formula is also satisfied when the geometric invariants of joints are considered as in the mechanical tolerancing process [18]. Thus, Clément defines Minimum Geometric Reference Set (MGRS) according to a joint degree of freedom [18]. For a single-axis rotation, the MGRS is a straight-line (i.e. the axis of rotation). 4 parameters are introduced for describing this geometric feature, i.e. 2 parameters to define the straight-line orientation and 2 parameters to define a point of this straight-line. For a unidirectional translation, the MGRS is a straightline parallel to the direction of the translation, i.e. 2 parameters to define the orientation of this line.

The problematic consists then to define a formalism which introduces a minimal number of parameters with regard the joint type, i.e. which introduces 4 parameters allowing to position and orient a rotational joint in space and 2 parameters for a prismatic joint. 
The objective of this article is, thus, to introduce a new geometric modeling approach of a serial robot (just with rotational joints in our case study). The introduced approach is based on the geometric joint invariants which are described by vectors. This approach is consistent with a classical identification process, the "Circle Point Analysis" (CPA). Indeed, this method directly identifies the invariants of rotational joints [10][6] (Figure 3). The consistency of the proposed geometric modeling method with the identification process naturally improves the final geometric accuracy of the studied robot.

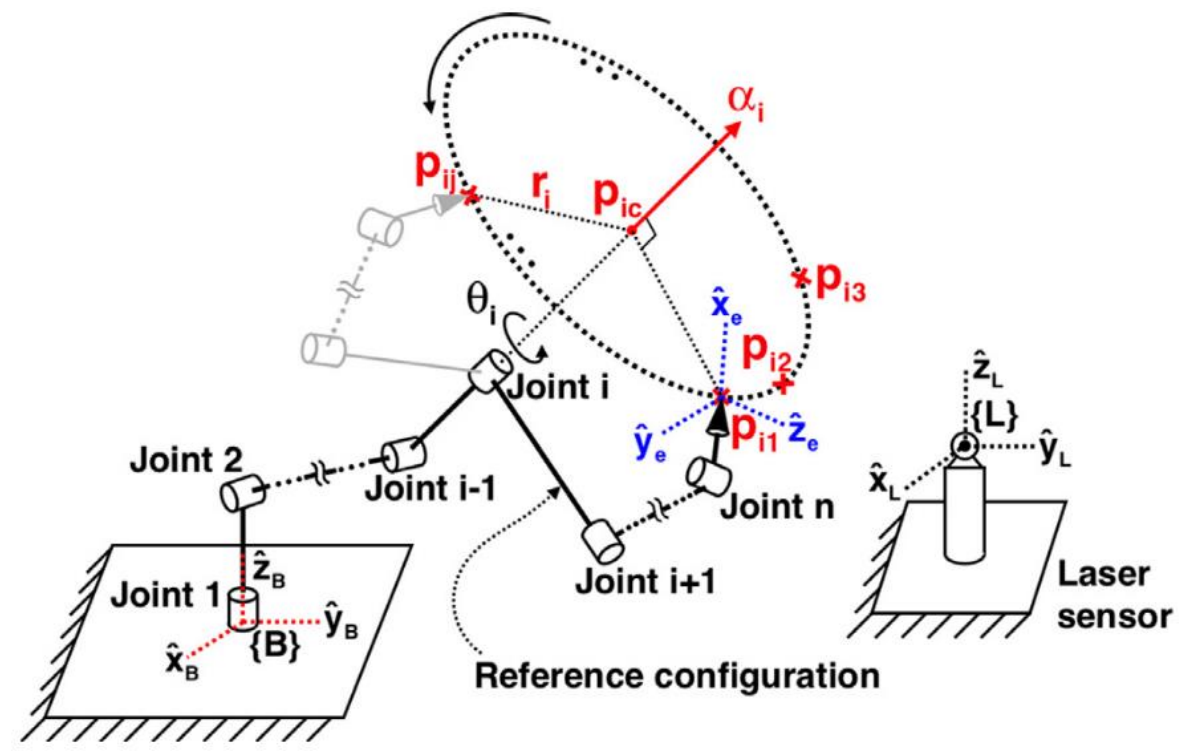

\section{Figure 3: Schematic diagram of the endpoint measurement of a robot manipulator for circular point analysis (CPA) when joint i rotates [6].}

The method presented in this article is illustrated on a SCARA (Selective Compliance Articulated Robot Arm) robot. Indeed, the choice of this simple robot ensures to describe clearly the introduced geometric modeling method and illustrates the accuracy benefit compare to the $\mathrm{DH}$ formalism, even if the system is simple and composed of two joints.

The article is organized as follows: First, the nominal model of the SCARA robot is introduced. Then, the method for modeling geometric invariant of a rotational joint is defined. Next, this modeling method and the identification process are applied to a SCARA robot. Finally, end-effector pose errors obtained after identification with our model and DH model are compared.

\section{Nominal modeling of a SCARA robot}

The aim of nominal modeling consists in defining a coordinate system fixed to each rigid part of the studied robot. The $\mathrm{DH}$ formalism is used to define the robot nominal model. This convention is commonly implemented and has shown is relevancy to define the nominal robot model [9].

In this paragraph, the $\mathrm{DH}$ convention is first introduced before presenting the studied SCARA robot and the application of the DH convention.

\subsection{Denavit-Hartenberg convention}

The DH convention parameters ensures to define the position, and the orientation of the coordinate systems fixed to each rigid part of a mechanism. Here, the aim is to define its geometric model [19]. 
Each rigid body $C_{i}$ of a mechanism is associated with a coordinate system $R_{i}\left(O_{i}, \vec{x}_{i}, \vec{y}_{i}, \vec{z}_{i}\right), i=0,1, \ldots, n$. The position and the orientation of coordinate systems $R_{1}$ to $R_{n-1}$ is defined according to specific rules which require 4 parameters $\left(d_{i}, \theta_{i}, a_{i}, \alpha_{i}\right)$ (Figure 1 ).

Frame $R_{i}$ is attached to each link $i$, such that [7] (Figure 1):

- the $\vec{z}_{i}$ axis is along the axis of joint $L_{i}$,

- the $\vec{x}_{i}$ axis is aligned with the common normal between $\vec{z}_{i}$ and $\vec{z}_{i+1}$,

- the intersection of $\vec{x}_{i}$ and $\vec{z}_{i}$ defines the origin $O_{i}$,

- the $\vec{y}_{i}$ axis is formed by the right-hand rule to complete the coordinate system

- $d_{i}$ is the distance between $\vec{x}_{i-1}$ and $\vec{x}_{i}$ along $\vec{z}_{i}$,

- $\quad \theta_{i}$ is the angle between $\vec{x}_{i-1}$ and $\vec{x}_{i}$,

- $\quad a_{i}$ is the distance between $\vec{z}_{i}$ and $\vec{z}_{i+1}$ along $\vec{x}_{i}$,

- $\quad \alpha_{i}$ is the angle between $\vec{z}_{i}$ and $\vec{z}_{i+1}$.

The positions and orientations of references $R_{0}$ and $R_{n}$ are chosen to minimize the number of introduced geometric parameters.

The transfromation from coordinate system $\mathrm{R}_{\mathrm{i}}$ to $\mathrm{R}_{\mathrm{i}+1}$ is then expressed from:

$$
{ }^{\mathbf{i}} \mathbf{T}_{\mathbf{i}+\mathbf{1}}=\left(\begin{array}{cccc}
1 & 0 & 0 & 0 \\
0 & 1 & 0 & 0 \\
0 & 0 & 1 & d_{i} \\
0 & 0 & 0 & 1
\end{array}\right)\left(\begin{array}{cccc}
\cos \left(\theta_{i}\right) & -\sin \left(\theta_{i}\right) & 0 & 0 \\
\sin \left(\theta_{i}\right) & \cos \left(\theta_{i}\right) & 0 & 0 \\
0 & 0 & 1 & 0 \\
0 & 0 & 0 & 1
\end{array}\right)\left(\begin{array}{llll}
1 & 0 & 0 & a_{i} \\
0 & 1 & 0 & 0 \\
0 & 0 & 1 & 0 \\
0 & 0 & 0 & 1
\end{array}\right)\left(\begin{array}{cccc}
1 & 0 & 0 & 0 \\
0 & \cos \left(\alpha_{i}\right) & -\sin \left(\alpha_{i}\right) & 0 \\
0 & \sin \left(\alpha_{i}\right) & \cos \left(\alpha_{i}\right) & 0 \\
0 & 0 & 0 & 1
\end{array}\right)
$$

$d_{i}, \theta_{i}, a_{i}$ and $\alpha_{i}$ are defined in Figure 1.

Thus, the nominal position of body $C_{n}$ in coordinate system $R_{0}$ is extracted from:

$$
{ }^{0} T_{n}={ }^{0} T_{1}{ }^{1} T_{2} \ldots{ }^{n-1} T_{n}
$$

\subsection{Application to the SCARA robot}

The first step of the DH convention application consists of setting up a coordinate system fixed to each robot rigid part (Figure 4). The position and orientation of the different coordinate systems allow defining the geometrical parameters necessary for the complete definition of the nominal model. These parameters are defined in Table 1 for the first two joints of a SCARA robot when considering $\vec{z}_{0}=\vec{z}_{1}$ and $O_{0}=O_{1}$. 


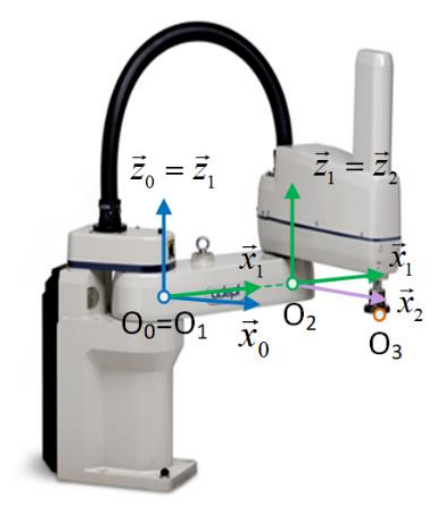

Figure 4: Coordinate systems associated with each robot rigid part.

\begin{tabular}{c|cccc}
\multicolumn{1}{c}{} & $d_{i}$ & $\theta_{i}$ & $a_{i}$ & $\alpha_{i}$ \\
\hline$R_{0} \rightarrow R_{1}$ & - & - & 0 & 0 \\
$R_{1} \rightarrow R_{2}$ & $d_{1}$ & $q_{1}$ & $a_{1}$ & 0 \\
$R_{2} \rightarrow R_{e}$ & 0 & $q_{2}$ & $a_{2}$ & 0
\end{tabular}

Table 1: DH geometric parameters of the studied SCARA robot.

The geometric parameters taken into account by the nominal geometric model of the first two joints of the SCARA robot are the lengths of the arms 1 and $2\left(a_{1}\right.$ and $\left.a_{2}\right)$, the offset $\mathrm{d}_{1}$ of the end-effector controlled point along the axis $\vec{z}_{2}$ as well as the joint coordinate value applied to the motors $\left(q_{1}, q_{2}\right)$.

\subsection{Definition of the SCARA robot nominal model}

The definition of the Direct Kinematic Model (DKM) is realized according to transformation matrices such as:

$$
\begin{aligned}
& { }^{\mathbf{0}} \mathbf{T}_{\mathbf{N}_{\mathbf{3}}}={ }^{\mathbf{0}} \mathbf{T}_{\mathbf{N}_{\mathbf{1}}}{ }^{\mathbf{1}} \mathbf{T}_{\mathbf{N}_{\mathbf{2}}}{ }^{{ }^{2}} \mathbf{T}_{\mathbf{N}_{\mathbf{3}}}= \\
& {\left[\begin{array}{cccc}
\cos \left(q_{1}+q_{2}\right) & -\sin \left(q_{1}+q_{2}\right) & 0 & a_{2} \cos \left(q_{1}+q_{2}\right)+a_{1} \cos \left(q_{1}\right) \\
\sin \left(q_{1}+q_{2}\right) & \cos \left(q_{1}+q_{2}\right) & 0 & a_{2} \sin \left(q_{1}+q_{2}\right)+a_{1} \sin \left(q_{1}\right) \\
0 & 0 & 1 & d_{1} \\
0 & 0 & 0 & 1
\end{array}\right]}
\end{aligned}
$$
system $\mathrm{R}_{\mathrm{j}}$.

where ${ }^{j} \mathbf{T}_{\mathbf{N}_{\boldsymbol{i}}}$ is the nominal transformation matrix of coordinate system $\mathrm{R}_{\mathrm{i}}$ to coordinate

The last column of this transformation matrix directly provides the coordinates of the nominal position of the point $\mathrm{O}_{3}$ in frame $\mathrm{R}_{0}$ :

$$
\overrightarrow{O_{1} O_{N_{3}}}=\left[\begin{array}{c}
{ }^{\mathbf{0}} \mathbf{T}_{\mathbf{N}_{\mathbf{3}}}(1,4) \\
{ }^{\mathbf{0}} \mathbf{T}_{\mathbf{N}_{\mathbf{3}}}(2,4) \\
{ }^{\mathbf{0}} \mathbf{T}_{\mathbf{N}_{\mathbf{3}}}(3,4)
\end{array}\right]_{R_{0}}=\left[\begin{array}{c}
a_{2} \cos \left(q_{1}+q_{2}\right)+a_{1} \cos \left(q_{1}\right) \\
a_{2} \sin \left(q_{1}+q_{2}\right)+a_{1} \sin \left(q_{1}\right) \\
d_{1}
\end{array}\right]_{R_{0}}
$$

This last expression gives the definition of the nominal DKM of the SCARA robot.

However, this formalism does not allow integrating orientation defects of the axis $\vec{z}_{i}$ in a plane different from the plane $\left(\vec{y}_{i-1}, \vec{z}_{i-1}\right)$. The introduction of a complete description of the 
orientation of a coordinate system regarding another requires the use of a more complete formalism. The following section introduces such formalism.

\section{Geometric defects introduction with a vector method}

The geometric behavior of a robot relies on the position and orientation of its joints. For the SCARA robot, introduced defects are linked to the orientation and position of robot rotational joints. The invariant of the rotational joint is a straight-line (rotational joint axis) [18]. Therefore, 4 parameters are introduced for describing this feature, i.e. 2 parameters to define the straight-line orientation and 2 parameters to define a point of this straight-line. In this way, the definition of the direct kinematic model of the SCARA robot requires the identification of 4 parameters, for each rotational joint, which describe the position and orientation of each rotational joint axis in the coordinate system associated with the previous joint axis (Figure 5). Note that, for a prismatic joint just the 2 parameters which define the straight-line orientation are necessary.

The following paragraph introduces the methodology proposed to define these 4 parameters.

$\left(O_{i-1}, \vec{z}_{i-1}\right)$ is the axis of rotational joint $\mathrm{i}-1$ and $\left(O_{i}, \vec{z}_{i}\right)$ the axis of rotational joint $i$. $O_{i}$ is the intersection point between plan $\left(O_{i-1}, \vec{x}_{i-1}, \vec{y}_{i-1}\right)$ and the axis of rotational joint $i$. $\vec{x}_{i-1}{ }^{\prime}$ is the direction vector of the straight line $\left(O_{i-1} O_{i}\right)$, thus $\overrightarrow{O_{i-1} O_{i}}=a_{i-1} \vec{x}_{i-1}{ }^{\prime}$. Note the scalar product $\vec{x}_{i-1} \cdot \vec{x}_{i-1}{ }^{\prime}=\cos \left(\theta_{i-1}\right)$. The coordinate system $R_{i-1}{ }^{\prime}$ is then defined from the cross product $\vec{y}_{i-1}{ }^{\prime}=\vec{z}_{i-1} \times \vec{x}_{i-1}{ }^{\prime} \cdot \vec{x}_{i}$ is along the projection of $\vec{x}_{i-1}{ }^{\prime}$ in the plan normal to $\vec{z}_{i}$ which contains $O_{i}$. Then the coordinate system $R_{i}$ is defined as $\vec{x}_{i-1}{ }^{\prime} \cdot \vec{y}_{i}=0, \vec{x}_{i} \cdot \vec{z}_{i}=0$ and $\vec{y}_{i}=\vec{z}_{i} \times \vec{x}_{i}$

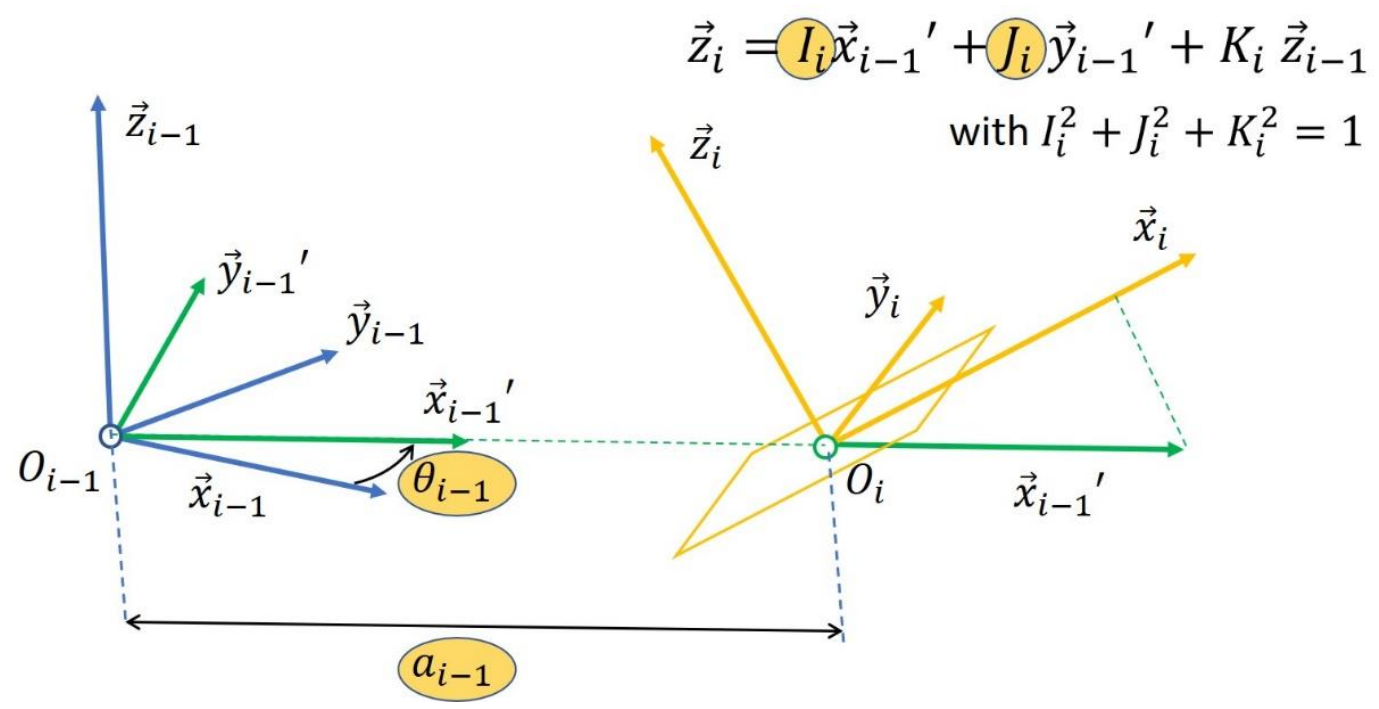

Figure 5: Invariant definition of two rotational joints of a robot arm.

Thus, the rotation matrix allowing defining the orientation of coordinate system $R_{i-1}{ }^{\prime}$ in coordinate system $R_{i-1}$ can be written as:

$$
{ }^{\mathbf{i}-\mathbf{1}} \mathbf{R}_{\mathbf{i}-\mathbf{1}},=\left(\begin{array}{ccc}
\cos \left(\theta_{i-1}\right) & -\sin \left(\theta_{i-1}\right) & 0 \\
\sin \left(\theta_{i-1}\right) & \cos \left(\theta_{i-1}\right) & 0 \\
0 & 0 & 1
\end{array}\right)
$$

with $\theta_{i-1}$ the angle between $\vec{x}_{i-1}$ and $\vec{x}_{i-1}{ }^{\prime}$. 
The rotation matrix allowing the expression of coordinate system $R_{i}$ relatively to coordinate system $R_{i-1}{ }^{\prime}$ is:

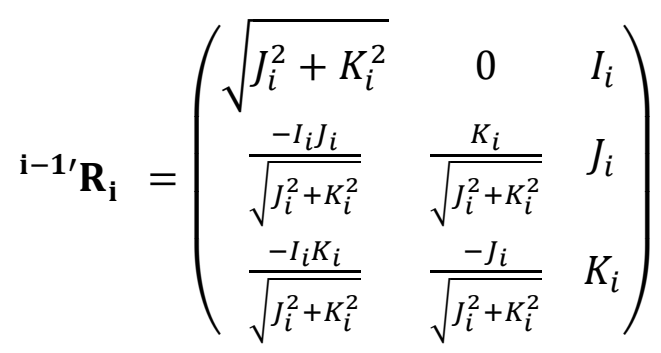

with $\vec{z}_{i}=I_{i} \vec{x}_{i-1}{ }^{\prime}+J_{i} \vec{y}_{i-1}{ }^{\prime}+K_{i} \vec{z}_{i-1}{ }^{\prime}$, i.e. $\left(I_{i}, J_{i}, K_{i}\right)$ are the coordinates of $\vec{z}_{i}$ in frame $R_{i-1}{ }^{\prime}$, with $\vec{x}_{i-1}^{\prime} \cdot \vec{y}_{i}=0, \vec{x}_{i} \cdot \vec{z}_{i}=0$ and $\vec{y}_{i}=\vec{z}_{i} \times \vec{x}_{i}$.

Thus, the identification of the position and the orientation of each rotational joint axis (a straight-line orientation and a point) in the coordinate system associated with the previous axis is performed thanks to 4 independent parameters $\theta_{i}, a_{i}, I_{i}, J_{i}$ and a dependent parameter $K_{i}=\sqrt{1-\left(I_{i}^{2}+J_{i}^{2}\right)}$. As $K_{i}$ is always positive, its imposes the direction of the unit vector of the joint axis. Note that, for a prismatic joint, this formalism is used by canceling $\theta_{i}$ and $a_{i}$.

The introduced parameters are different from those defined with the DH convention. Indeed, with our methodology, $\vec{z}_{i}$ axis is not constrained to be in the plane $\left(\vec{y}_{i-1}, \vec{z}_{i-1}\right)$. Moreover, $R_{i}$ position and orientation are not constrained by the definition of coordinate system $R_{i-1}$.

\section{Application to the geometric behavior modeling of the SCARA robot}

Applying the strategy previously presented to the first two axes of a SCARA robot, the model presented in Figure 6 is obtained.

First, $\vec{z}_{1}$ and $\vec{z}_{2}$ are chosen along the rotation axis of the first two rotational joints of a SCARA robot. Point $O_{1}$ is arbitrary chosen on the first rotational joint axis. For our study, we consider that $R_{1}$ is the robot coordinate system. Coordinate system $R_{2}\left(O_{2}{ }^{\prime}, \vec{x}_{2}, \vec{y}_{2}, \vec{z}_{2}\right)$ is defined according to the methodology presented in section 3. Since the studied system features only 2 degrees of freedom, a coordinate system $R_{3}$ is not introduced, but only a point $O_{3}$ which corresponds to the end-effector control point. The position of this point $O_{3}$ is defined accordingly to the one of point $O_{i}$ in section 3 and the introduction of a new point $O_{2}$. The introduction of this new point $O_{2}$ let open the possibility of an offset between chosen point $O_{1}$ and end-effector control point $O_{3} . O_{1}$ corresponds to a virtual point and, end-effector control point $O_{3}$ can be physically defined and can change accordingly to the used end-effector. Finally, 7 parameters are introduced: $\theta_{1}, \theta_{2}, a_{1}, a_{2}, d_{2}, I_{2}, J_{2}$. 


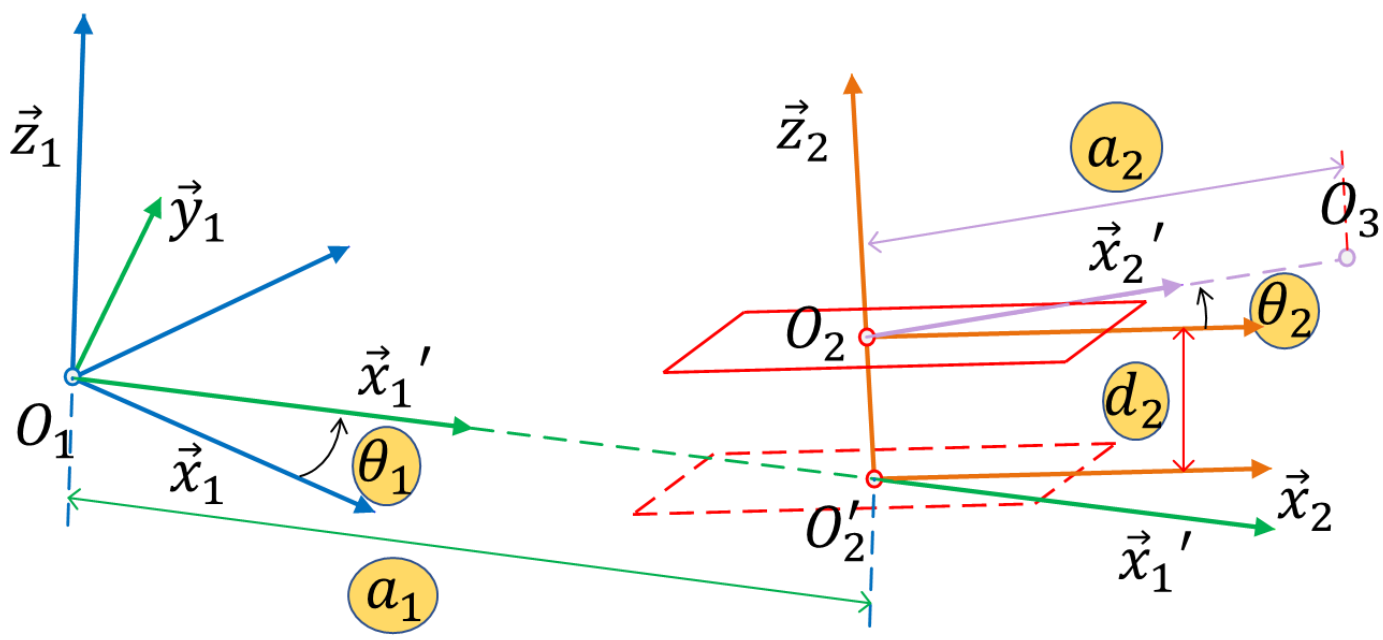

$R_{1}=\left(O_{1}, \vec{x}_{1}, \vec{y}_{1}, \vec{z}_{1}\right)$ is considered as the fixed coordinate system

Figure 6: Vector model of a SCARA robot.

The DKM is expressed as:

$$
\overrightarrow{O_{1} O_{3}}=\overrightarrow{O_{1} O_{2}^{\prime}}+\overrightarrow{O_{2}^{\prime} O_{2}}+\overrightarrow{O_{2} O_{3}}=a_{1} \vec{x}_{1}{ }^{\prime}+d_{2} \vec{Z}_{2}+a_{2} \vec{x}_{2}{ }^{\prime}
$$

With $\quad \vec{x}_{1}{ }^{\prime}=\left(\begin{array}{ccc}\cos \left(q_{1}+d \theta_{1}\right) & -\sin \left(q_{1}+d \theta_{1}\right) & 0 \\ \sin \left(q_{1}+d \theta_{1}\right) & \cos \left(q_{1}+d \theta_{1}\right) & 0 \\ 0 & 0 & 1\end{array}\right)\left(\begin{array}{l}1 \\ 0 \\ 0\end{array}\right)={ }^{\mathbf{0}} \mathbf{R}_{\mathbf{1}}\left(\begin{array}{l}1 \\ 0 \\ 0\end{array}\right), \quad \vec{x}_{2}{ }^{\prime}=$ $\cos \left(q_{2}+d \theta_{2}\right) \vec{x}_{2}+\sin \left(q_{2}+d \theta_{2}\right) \vec{y}_{2}, q_{1}$ and $q_{2}$ are actuated motor values, and $d \theta_{1}$ and $d \theta_{2}$ are actuated motor offsets such as $\theta_{1}=q_{1}+d \theta_{1}$ and $\theta_{2}=q_{2}+d \theta_{2}$.

Let $\left(I_{2}, J_{2}, K_{2}\right)$ be coordinates of $\vec{z}_{2}$ in the coordinate system $R_{1}{ }^{\prime}$ :

$$
{ }^{1} \mathbf{R}_{2}=\left(\begin{array}{ccc}
\sqrt{J_{2}^{2}+K_{2}^{2}} & 0 & I_{2} \\
\frac{-I_{2} J_{2}}{\sqrt{J_{2}^{2}+K_{2}^{2}}} & \frac{K_{2}}{\sqrt{J_{2}^{2}+K_{2}^{2}}} & J_{2} \\
\frac{-I_{2} K_{2}}{\sqrt{J_{2}^{2}+K_{2}^{2}}} & \frac{-J_{2}}{\sqrt{J_{2}^{2}+K_{2}^{2}}} & K_{2}
\end{array}\right)
$$
$R_{1}{ }^{\prime}$

This matrix ${ }^{\mathbf{1}} \mathbf{R}_{\mathbf{2}}$ defines the orientation of coordinate system $R_{2}$ in coordinate system

Note:

$$
{ }^{2} \mathbf{R}_{2^{\prime}}=\left(\begin{array}{ccc}
\cos \left(q_{2}+d \theta_{2}\right) & -\sin \left(q_{2}+d \theta_{2}\right) & 0 \\
\sin \left(q_{2}+d \theta_{2}\right) & \cos \left(q_{2}+d \theta_{2}\right) & 0 \\
0 & 0 & 1
\end{array}\right)
$$

Then, SCARA DKM is expressed by:

$$
\overrightarrow{O_{1} O_{3}}=a_{1}{ }^{1} \mathbf{R}_{1},\left(\begin{array}{l}
1 \\
0 \\
0
\end{array}\right)+d_{2}{ }^{1} \mathbf{R}_{1},{ }^{1} \mathbf{R}_{\mathbf{2}}\left(\begin{array}{l}
0 \\
0 \\
1
\end{array}\right)+a_{2}{ }^{1} \mathbf{R}_{\mathbf{1}},{ }^{1} \mathbf{R}_{\mathbf{2}}{ }^{2} \mathbf{R}_{\mathbf{2}}\left(\begin{array}{l}
1 \\
0 \\
0
\end{array}\right)
$$

The parameters to be identified are $a_{1}, d_{2}, a_{2}, d \theta_{1}, d \theta_{2}$ and the $I_{2}$ and $J_{2}$ coordinates of $\vec{Z}_{2}$ in $R_{1}{ }^{\prime}$. 6 parameters should be added to position and orient coordinate system 
$R_{1}\left(O_{1}, \vec{x}_{1}, \vec{y}_{1}, \vec{z}_{1}\right)$ which is coincident with the robot base coordinate system in the measurement coordinate system.

\section{Geometric parameters identification}

The identification process is realized with a laser tracker (Leica AT-901) with an uncertainty of $10 \mu \mathrm{m}+5 \mu \mathrm{m} / \mathrm{m}$ (Figure 7). The target is localized at the extremity of the endeffector. The studied robot joints are moved with harmonic drives. These drives have low backlash [20]. Thus, the influence of backlash in identification process is neglected.

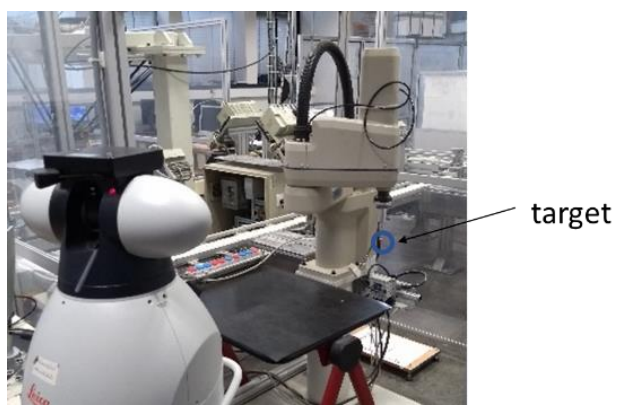

Figure 7: Target measurement with a laser tracker on a SCARA robot.

To directly identify the invariants of robot joints from the laser tracker measurements, the CPA method is used [21][6]. This method ensures to identify each robot rotational joint axis and is described in [21]. This method consists in measuring the tool path followed by the endeffector during a single actuated joint movement (Figure 8). The theoretical tool path is a circle. The normal vector of the plane in which the circle movement lies is the axis vector of the actuated joint, and the circle center is a point of this joint axis. For a robot with $N$ degrees of freedom, $N$ circles should be measured. To minimize the regression errors, the joint positions should be uniformly spaced [21]. The identification accuracy depends on the ability to trace the target on a portion of the total joint travel as large as possible according to the measurement mean visibility [21]. For each value of the joint position, the coordinate of the target is measured in the laser tracker coordinate system. To avoid dynamic phenomena, the robot is stopped during each measurement.

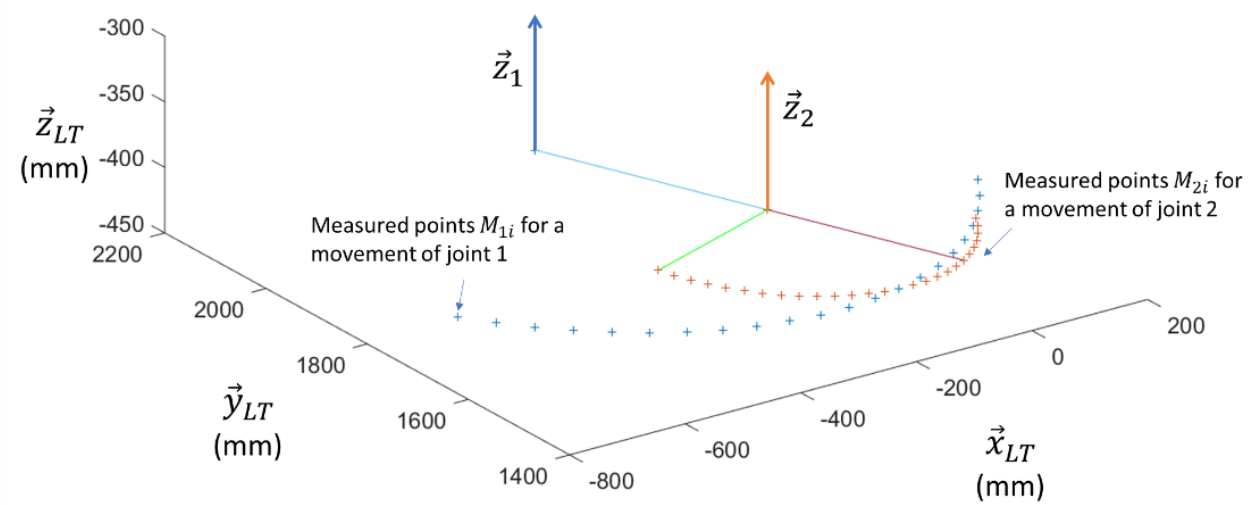

Figure 8: Measured points in the laser tracker coordinate system during a movement of joint 1 and joint 2 .

As shown on Figure 8, points $\mathbf{M}_{\mathbf{1}}$ and $\mathbf{M}_{\mathbf{2}}$ are the different positions reached by the target during a rotational movement, respectively, of axis 1 or axis 2 . The coordinates of the 
points measured in laser tracker coordinate system $R_{L T}\left(O_{L T}, \vec{x}_{L T}, \vec{y}_{L T}, \vec{z}_{L T}\right)$ are given in appendix A.

In appendix A, we can see that seven positions are measured twice. Between the two measurements, there is a repositioning of the laser tracker target. From these measurements, a maximum target repositioning defect of $0.032 \mathrm{~mm}$ is obtained. This error will affecte the identification process.

In this paper, the coordinates of a vector $\vec{x}$ on a given coordinate system $R_{i}$ is noted $\vec{x}^{R_{i}}$.

The geometric parameters of the SCARA robot are identified from two geometric construction steps:

- From measured points $\mathbf{M}_{\mathbf{1}}$ obtained during a movement of joint 1, the leastsquares plan $\mathcal{P}_{1}$ is computed. The normal of this plan ensures to identify the coordinate of $\vec{z}_{1}^{R_{L T}}$ in the laser tracker coordinate system (Figure 4). The set of points $\mathbf{M}_{1}$ are projected in plane $\mathcal{P}_{1}$. The least-square circle $\mathcal{C}_{1}$ is then computed from these projected points $\mathbf{M}_{1 \mathbf{p}}$. The center of circle $\mathcal{C}_{1}$ allows identifying the coordinates of point $O_{1}^{R_{L T}}$ in the laser tracker coordinate system (Figure 4).

- From measured points $\mathbf{M}_{2}$ obtained during a movement of joint 2, the leastsquares plan $\mathcal{P}_{2}$ is computed. The normal of this plan ensure to identify the coordinate of $\vec{z}_{2}^{R} L T$ in laser tracker coordinate system (Figure 4). The set of points $\mathbf{M}_{2}$ are projected in plane $\mathcal{P}_{2}$. The least-square circle $\mathcal{C}_{2}$ is then computed from these projected points $\mathbf{M}_{2 \mathbf{p}}$. The center of circle $\mathcal{C}_{2}$ allows identifying the coordinates of point $O_{2}^{R_{L T}}$ in the laser tracker coordinate system (Figure 4).

From these geometric constructions, the geometric parameters introduced in section 4 are identified. In this identification process, we consider that the base coordinate system of the robot is coincident with $R_{1}$, i.e. $R_{1}$ is the fixed coordinate system. The orientation of $\vec{x}_{1}$ is aligned with $\vec{x}_{1}{ }^{\prime}$ when $q_{1}=0$, i.e. $d \theta_{1}=0$. The value of $d \theta_{2}$ is the angle between $\vec{x}_{2}$ and $\vec{x}_{2}{ }^{\prime}$ when $q_{2}=0 . d \theta_{2}$ is obtained from the angle between $\vec{x}_{2}$ and $\overrightarrow{O_{2} M_{2 p}}$ when $q_{1}=0$.

$a_{2}$ is the radius of circle $\mathcal{C}_{2} . O_{2}^{\prime}$ is the intersection point between the straight-line $\left(\mathrm{O}_{2}, \vec{z}_{2}\right)$ and plane $\mathcal{P}_{1} . d_{2}$ is such that $\overrightarrow{O_{2}^{\prime} O_{2}}=\overrightarrow{O_{1} O_{2}}-\overrightarrow{O_{1} O_{2}^{\prime}}=d_{2} \vec{Z}_{2}$. However, $\overrightarrow{O_{1} O_{2}^{\prime}} \cdot \vec{z}_{1}=0$. Thus, $d_{2}$ is computed from:

$$
d_{2}=\frac{\overrightarrow{O_{1} O_{2}} \cdot \vec{z}_{1}}{\vec{z}_{1} \cdot \vec{z}_{2}}
$$

$a_{1}$ is the distance between point $O_{1}$ and point $O_{2}^{\prime}$ with $a_{1}=\left\|\overrightarrow{O_{1} O_{2}^{\prime}}\right\|$. Moreover, $\overrightarrow{O_{1} O_{2}^{\prime}}=$ $\overrightarrow{O_{1} O_{2}}-d_{2} \vec{z}_{2}$. The determination of $\vec{x}_{1}{ }^{\prime}$ comes from:

$$
\vec{x}_{1}^{\prime}=\frac{\overrightarrow{o_{1} O_{2}^{\prime}}}{a_{1}}
$$

Thus, $\vec{y}_{1}{ }^{\prime}=\vec{z}_{1} \times \vec{x}_{1}{ }^{\prime}$. We can then deduce $I_{2}=\vec{z}_{2} \cdot \vec{x}_{1}{ }^{\prime}, J_{2}=\vec{z}_{2} \cdot \vec{y}_{1}{ }^{\prime}$ and $K_{2}=\vec{z}_{2} \cdot \vec{z}_{1}$.

Finally, $\vec{x}_{1}=\vec{x}_{1}{ }^{\prime}$ when $q_{1}=0$ and $\vec{y}_{1}=\vec{z}_{1} \times \vec{x}_{1}$. Note that coordinate system $R_{1}$ can be identified completely only at the end of the numerical process.

The transformation matrix which expresses coordinate system $R_{1}$ in laser tracker coordinate system is finally defined such as: 


$$
{ }^{\mathbf{L T}} \mathbf{T}_{\mathbf{R}_{\mathbf{1}}}=\left[\begin{array}{cccc}
\vec{x}_{1}^{R_{L T}} & \vec{y}_{1}^{R_{L T}} & \vec{z}_{1}^{R_{L T}} & O_{1}^{R_{L T}} \\
0 & 0 & 0 & 1
\end{array}\right]
$$

The identified values are given in Table 2 .

\begin{tabular}{|c|c|c|c|c|}
\hline Geometric & \multicolumn{4}{|c|}{ Identified values } \\
\hline${ }^{\mathbf{R}_{\mathrm{LT}}} \mathbf{T}_{\mathbf{R}_{\mathbf{1}}}$ & {$\left[\begin{array}{c}-0.383553 \\
-0.923519 \\
0.000415 \\
0\end{array}\right.$} & $\begin{array}{c}0.923472 \\
-0.383539 \\
-0.009853 \\
0\end{array}$ & $\begin{array}{c}0.009258 \\
-0.003396 \\
0.999951 \\
0\end{array}$ & $\left.\begin{array}{c}-295.393 \mathrm{~mm} \\
2044.593 \mathrm{~mm} \\
-413.640 \mathrm{~mm} \\
1\end{array}\right]$ \\
\hline$\vec{Z}_{2}^{R_{1^{\prime}}}$ & \multicolumn{4}{|c|}{$\begin{array}{r}I_{2}=0.000105 \\
J_{2}=0.000114 \\
K_{2}=0.999999\end{array}$} \\
\hline$a_{1}$ & \multicolumn{4}{|c|}{$325.034 \mathrm{~mm}$} \\
\hline$a_{2}$ & \multicolumn{4}{|c|}{$274.199 \mathrm{~mm}$} \\
\hline$d_{2}$ & \multicolumn{4}{|c|}{$0.056 \mathrm{~mm}$} \\
\hline$d \theta_{1}$ & \multicolumn{4}{|c|}{$0 \mathrm{rad}$} \\
\hline$d \theta_{2}$ & \multicolumn{4}{|c|}{$0.000279 \mathrm{rad}$} \\
\hline
\end{tabular}

Table 2: Identified geometric parameters

The residual errors after identification for points $\mathbf{M}_{\mathbf{1}}$ and $\mathbf{M}_{\mathbf{2}}$ are given in Figure 9 . The maximum residual error value is obtained for a movement of joint 1 and is near $0.069 \mathrm{~mm}$. The mean residual error for a joint 1 movement is $0.030 \mathrm{~mm}$ and $0.015 \mathrm{~mm}$ for a joint 2 movement. All these values are close to the maximum observed target repositioning defect which is 0.032 $\mathrm{mm}$. 

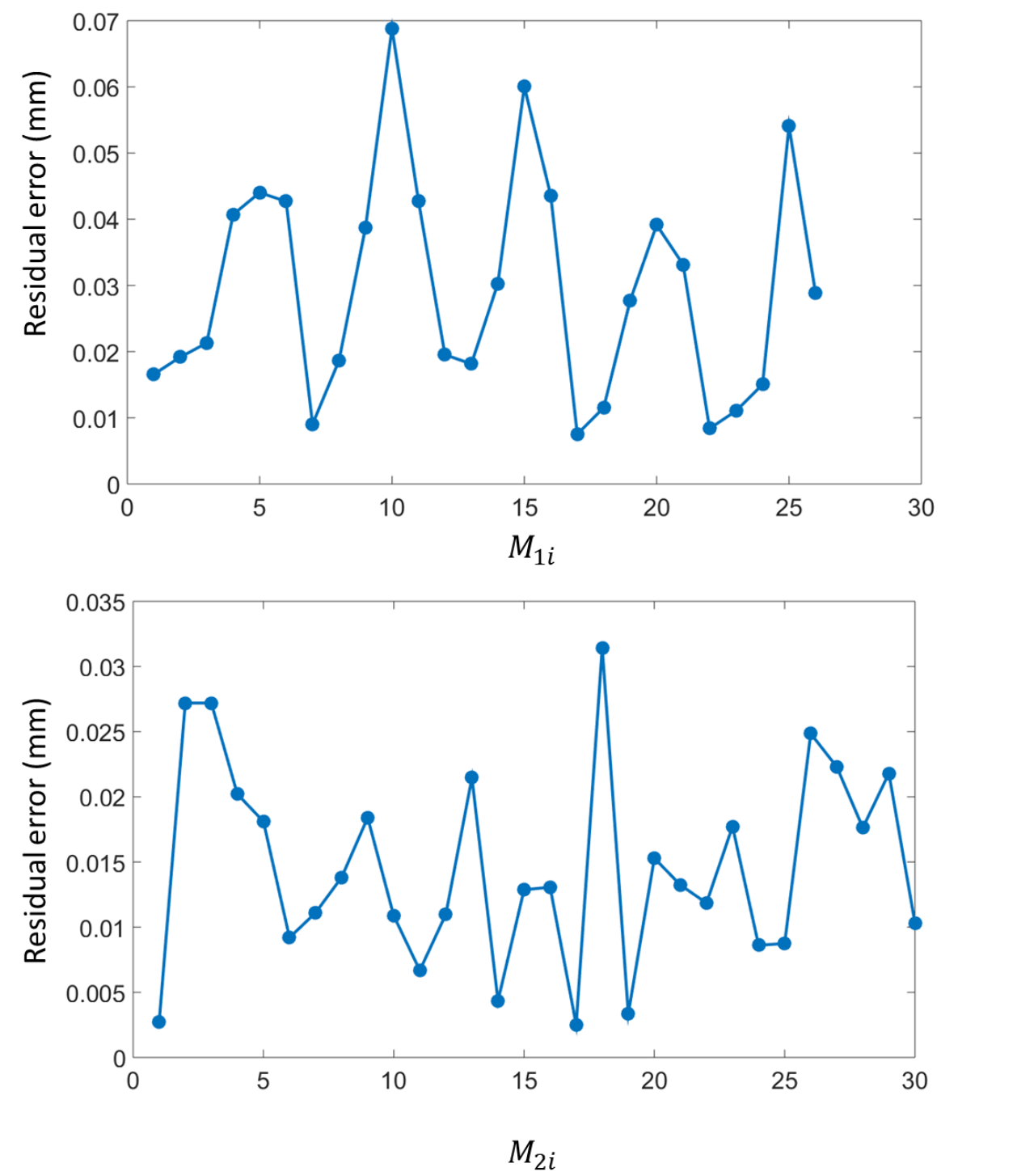

Figure 9: Residual errors on points used for the identification process

From the identification process, the flatness and roundness of each joint are estimated. These features are technical behavior which characterize the geometric joint behavior (Figure 10). The flatness is estimated by computing the distance between each measured point and the associated least-square plane. The roundness correspond to the distance between each measured point projection and the associated least-square circle. For joint 1 , the flatness defect is 0.03 $\mathrm{mm}$ and the roundness defect is $0.031 \mathrm{~mm}$. For joint 2 , the flatness defect is $0.013 \mathrm{~mm}$ and the roundness defect is $0.018 \mathrm{~mm}$. 

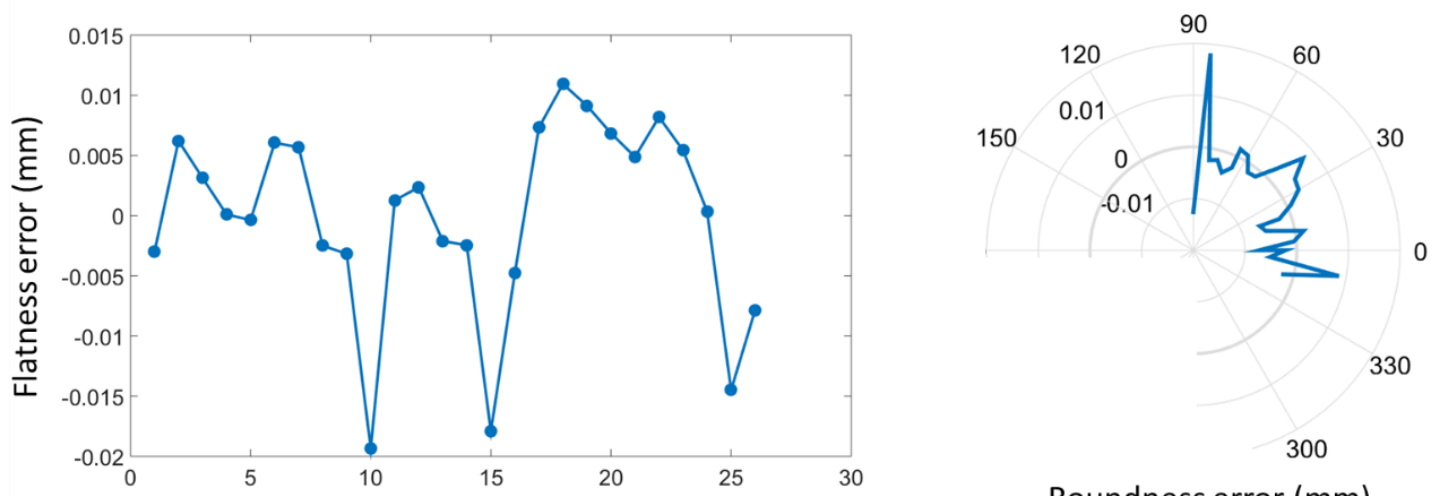

Roundness error ( $\mathrm{mm}$ )

\section{Geometric defect of joint 1}
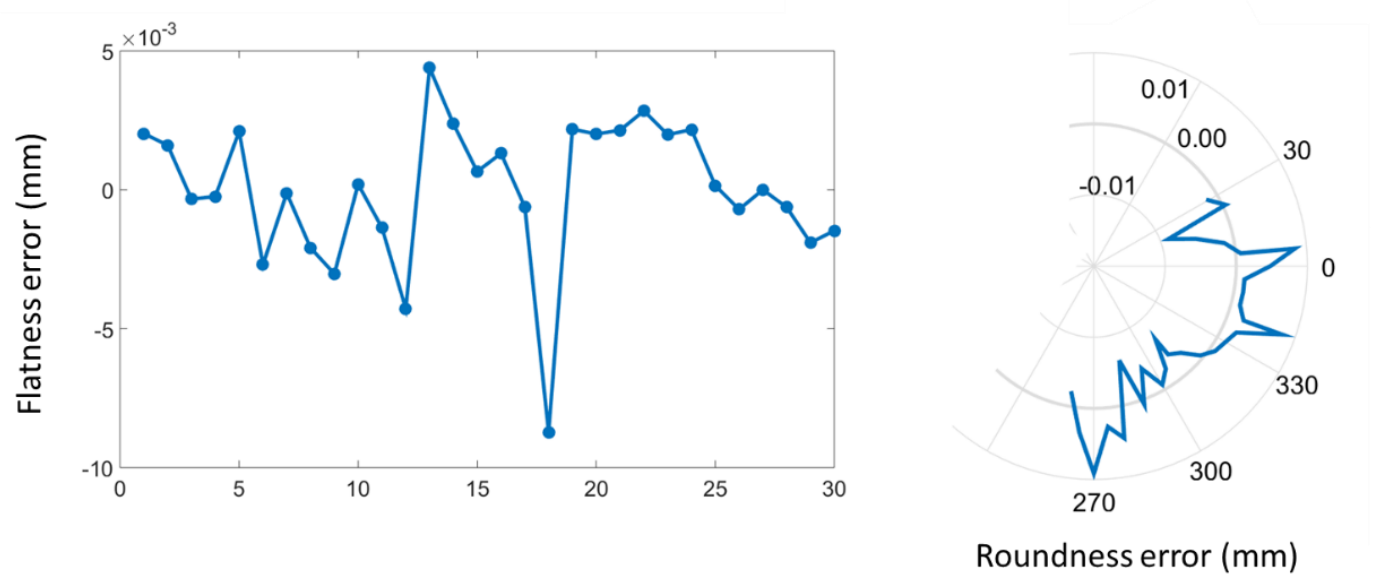

Geometric defect of joint 2

Figure 10: Geometrical defect of joint 1 and joint 2.

Nine supplementary points $\mathbf{M}_{\mathbf{v}}$ are measured with the laser tracker for validating realized identification. The coordinates of these points are given in Appendix B. The errors between measured point positions and simulated with identified DKM are less than $0.046 \mathrm{~mm}$ (Figure 11). This maximum error is less than the maximum residual error of the identification process which is $0.069 \mathrm{~mm}$ in our case study. The inaccuracy of the measurement with the laser tracker, the repeatability of the measurement process, the repeatability of the robot, and the uncompensated defect like joint flatness and roundness explain this error. 


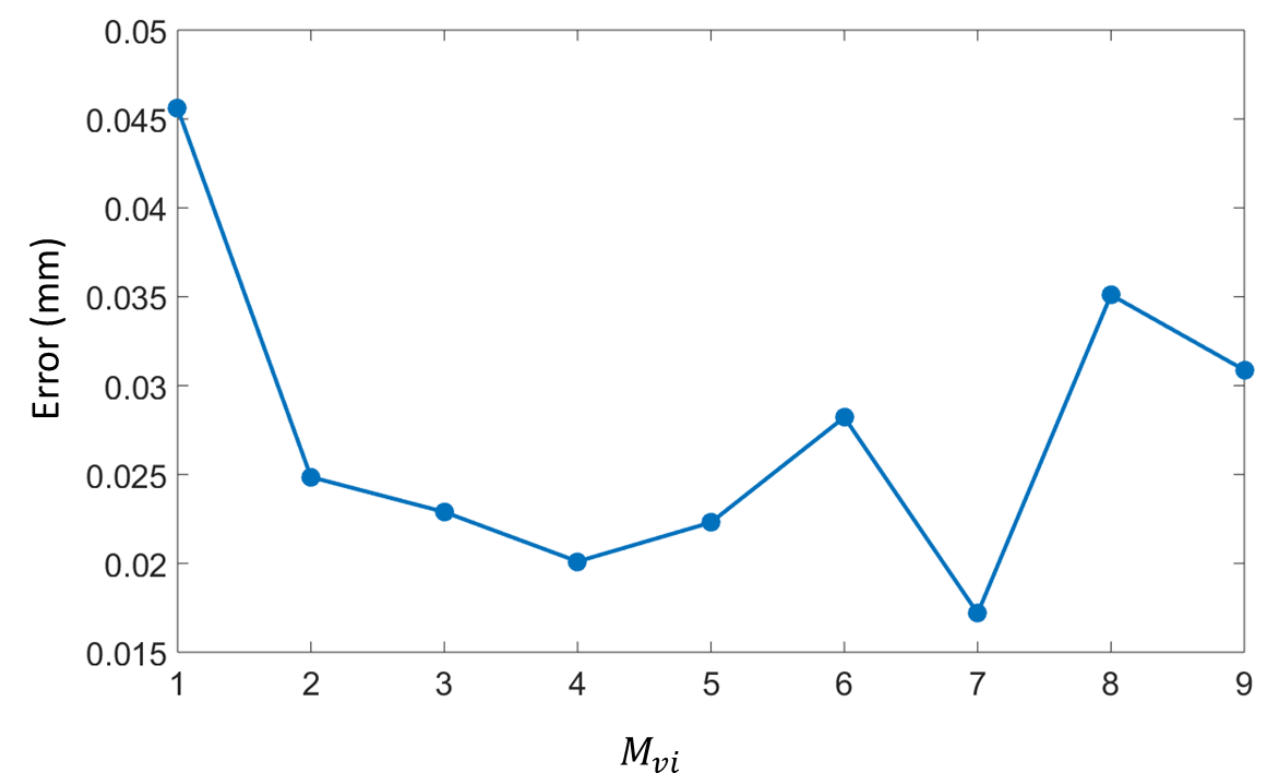

Figure 11: Error between the measured position points and the simulated with identified DKM

To illustrate the benefit of the new vector modeling method introduced in this paper on the final accuracy of geometric model, a comparison between these results and those obtained after an identification of a DH SCARA identified DKM is realized in the following section.

\section{Identification of the DH SCARA geometric model}

The identification of the DH SCARA geometric model is realized with two viewpoints. The first one brings to realize an identification of just the first order defect (dimensional defect), i.e. $a_{1}, d_{2}$, and $a_{2}$. The second one introduces second-order defects which are considered as small displacement. The DH geometric parameters become as in Table 3 if the base coordinate system of the robot is coincident with $R_{1}$, and $R_{2}{ }^{\prime}$ and $R_{e}$ have the same unit vectors. Thus, two second-order defects are introduced: $d \alpha_{2}$ and $d \theta_{2}$.

\begin{tabular}{c|cccc} 
& $d_{i}$ & $\theta_{i}$ & $a_{i}$ & $\alpha_{i}$ \\
\hline$R_{1} \rightarrow R_{1}{ }^{\prime}$ & 0 & $\mathrm{q}_{1}$ & 0 & 0 \\
$R_{1}{ }^{\prime} \rightarrow R_{2}$ & 0 & 0 & $a_{1}$ & $d \alpha_{2}$ \\
$R_{2}{ }^{\prime} \rightarrow R_{2}$ & $d_{2}$ & $q_{2}+d \theta_{2}$ & $a_{2}$ & 0
\end{tabular}

Table 3: first and second order of DH geometric parameters.

In the following sections, the identification of DH SCARA geometric parameters with only first-order defects is firstly presented. Afterwards, we introduce the identification of DH SCARA geometric parameters with first-order and second-order defects.

\subsection{Identification of the DH SCARA geometric parameters reduced to first-order defects}

The identification process is realized from points $\mathbf{M}_{\mathbf{1}}$ and $\mathbf{M}_{\mathbf{2}}$ measurement. Thus, the position and orientation of coordinate system $R_{1}$ regarding laser tracker coordinate system is the same as in Table 2. All points $\mathbf{M}_{1}$ are projected in plane $\mathcal{P}_{1}$. The position of point $O_{2}$ is computed from a translation of plane $\mathcal{P}_{1}$ with a least square minimization regarding points $\mathbf{M}_{2}$.

The identified values are given in Table 4. 


\begin{tabular}{|c|cccc|}
\hline Geometric parameters & \multicolumn{4}{|c|}{ Identified values } \\
\hline $\mathbf{R}_{\mathbf{L T}} \mathbf{T}_{\mathbf{R}_{\mathbf{1}}}$ & {$\left[\begin{array}{cccc}-0.383553 & 0.923472 & 0.009258 & -295.393 \mathrm{~mm} \\
-0.923519 & -0.383539 & -0.003396 & 2044.593 \mathrm{~mm} \\
0.000415 & -0.009853 & 0.999951 & -413.640 \mathrm{~mm} \\
0 & 0 & 0 & 1\end{array}\right]$} \\
\hline$a_{1}$ & \multicolumn{4}{|c}{$325.034 \mathrm{~mm}$} \\
\hline$a_{2}$ & \multicolumn{4}{|c}{$0.022 \mathrm{~mm}$} \\
\hline$d_{2}$ & \multicolumn{4}{|c|}{$0.199 \mathrm{~mm}$} \\
\hline
\end{tabular}

Table 4: Geometric parameter identified values for DH SCARA first-order geometric model.

The residual errors after identification for points $\mathbf{M}_{\mathbf{1}}$ and $\mathbf{M}_{\mathbf{2}}$ are given in Figure 12. The maximum residual error value is obtained for a movement of joint 1 and is near $0.142 \mathrm{~mm}$. The mean residual error for a joint 1 movement is $0.103 \mathrm{~mm}$ and $0.070 \mathrm{~mm}$ for a joint 2 movement.
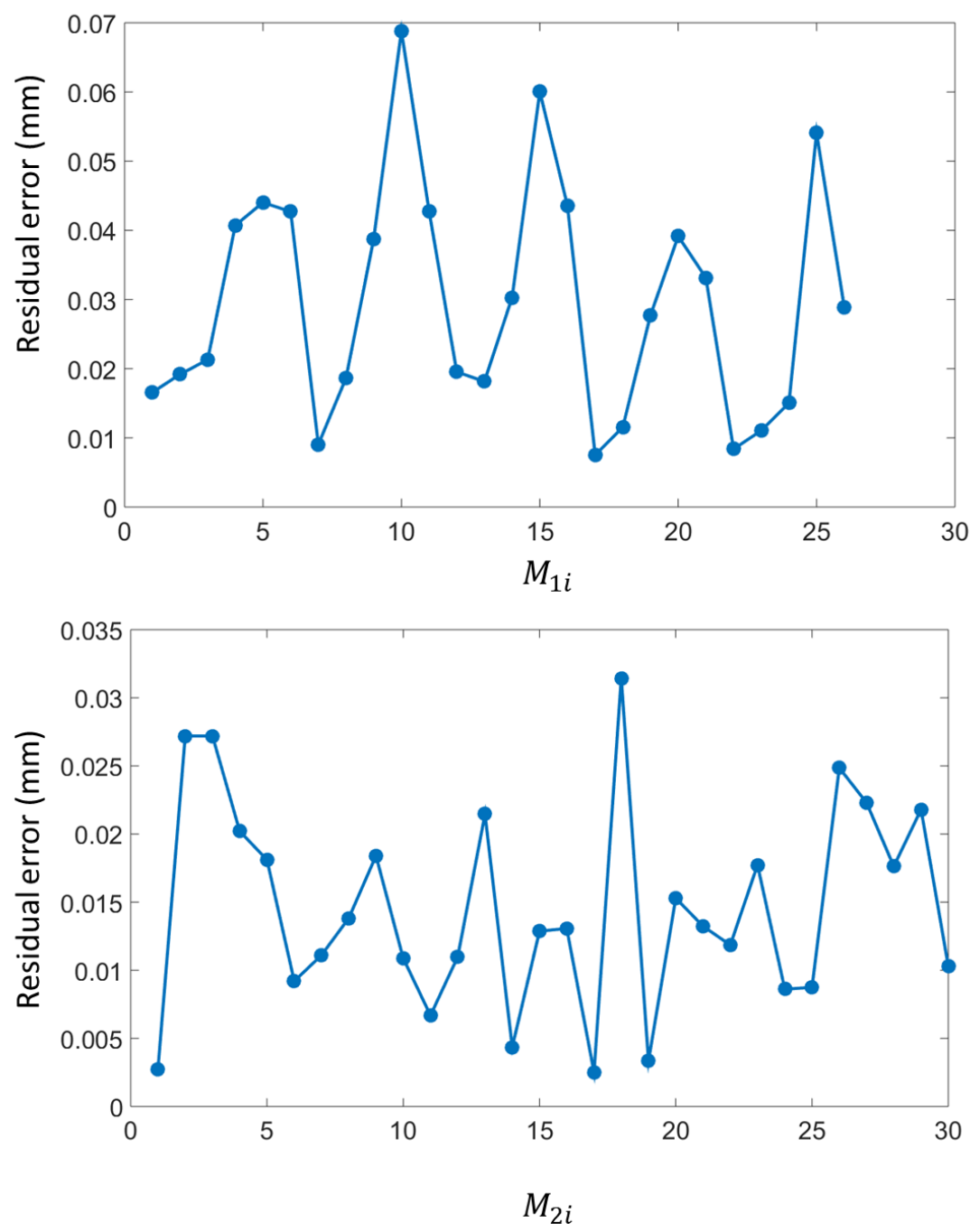

Figure 12: Residual errors for DH SCARA first-order geometric model.

The same nine validation points are used to estimate the accuracy benefit brings by the vector geometric model (Figure 13). The errors between measured points position and simulated with identified DKM are less than $0.122 \mathrm{~mm}$. The defect is multiplied by two compared to the proposed vector method. 


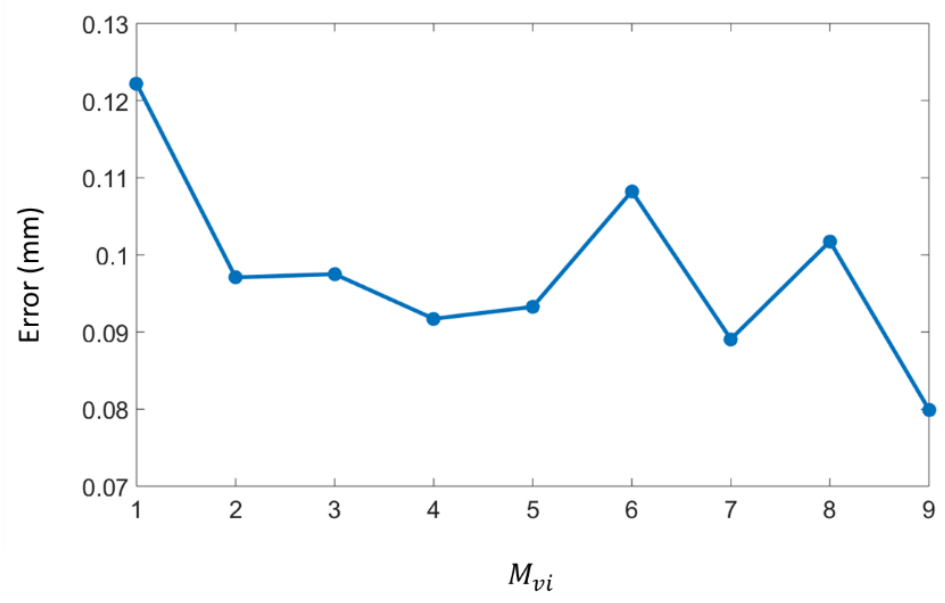

Figure 13: Error between measured position points and simulated with identified DH firstorder DKM.

However, in this DH model only the first-order defects are considered. In the following section, second-order defects are introduced to improve the final accuracy of the robot.

\subsection{Identification of the DH SCARA geometric parameters with first and second-order defects}

For the case of the DH geometric model with first and second-order defects, the identification process based on CPA cannot be simply implemented. Indeed, with these DH geometric parameters, the only orientation defect between axis $\vec{z}_{1}$ and $\vec{z}_{2}$ is a rotation $d \alpha_{2}$ around $\vec{x}_{1}{ }^{\prime}$. This constraint cannot be easily written in the measurement coordinate system.

In this case, the identification process should be realized by a minimization of a cost function expressed directly from the DKM.

Only matrix ${ }^{1 '} \mathbf{R}_{2}$ is modified:

$$
{ }^{1} \mathbf{R}_{2}=\left(\begin{array}{ccc}
1 & 0 & 0 \\
0 & \sqrt{1-d \alpha_{2}^{2}} & d \alpha_{2} \\
0 & -d \alpha_{2} & \sqrt{1-d \alpha_{2}^{2}}
\end{array}\right)
$$

The SCARA DKM is expressed from equation (12).

The geometric parameters of the SCARA robot are identified in two steps:

- The first step is like the one of section 5. From measured points $\mathbf{M}_{\mathbf{1}}$ obtained during a movement of joint 1 , the least-squares plan $\mathcal{P}_{1}$ and the least-square circle $\mathcal{C}_{1}$ are computed. The coordinate of $\vec{z}_{1}^{R_{L T}}$ and of point $O_{1}^{R_{L T}}$ in the laser tracker coordinate system are identified (Figure 4).

- The second step consists of the minimization of a cost function which expresses the difference between the measured coordinate of points $\mathbf{M}_{2}$ in the laser coordinate system with the computed vector $\mathbf{0}_{\mathbf{1}} \mathbf{0}_{\mathbf{3}}^{\mathbf{R}_{\mathbf{L T}}}=\left[{\overrightarrow{\mathrm{O}_{1} \mathrm{O}_{3}}}^{R_{L T}}\left(q_{2_{i}}\right)\right]$ :

$$
f_{\text {cost }}=\sqrt{\sum_{i=1}^{30}\left(\overrightarrow{O_{1} M_{2 i}}-{\overrightarrow{O_{1} O_{3}}}^{R}\left(q_{2_{i}}\right)\right)}
$$

The optimized parameters are the coordinate of $\vec{x}_{1}^{R_{L T}}, a_{1}, a_{2}, d_{2}, d \theta_{2}$ and $d \alpha_{2}$. 
The optimization numerical process is realized with the function "Isqnonlin" of Matlab®. The optimization stops due to the selected value of the step size tolerance which is $10^{-10}$.

The identified values are given in Table 5 .

\begin{tabular}{|c|c|c|c|c|}
\hline Geometric parameters & \multicolumn{4}{|c|}{ Identified values } \\
\hline \multirow{4}{*}{${ }^{\mathbf{R}_{\mathbf{L T}}} \mathbf{T}_{\mathbf{R}_{1}}$} & {$[-0.383541$} & 0.923478 & 0.009258 & $-295.393 \mathrm{~mm}$ \\
\hline & -0.923524 & -0.383525 & -0.003396 & $2044.593 \mathrm{~mm}$ \\
\hline & 0.000279 & -0.009853 & 0.999951 & $-413.640 \mathrm{~mm}$ \\
\hline & 0 & 0 & 0 & \\
\hline$a_{1}$ & \multicolumn{4}{|c|}{$325.033 \mathrm{~mm}$} \\
\hline$a_{2}$ & \multicolumn{4}{|c|}{$274.204 \mathrm{~mm}$} \\
\hline$d_{2}$ & \multicolumn{4}{|c|}{$0.046 \mathrm{~mm}$} \\
\hline$d \theta_{2}$ & \multicolumn{4}{|c|}{$0.000214 \mathrm{rad}$} \\
\hline$d \alpha_{2}$ & \multicolumn{4}{|c|}{$0.000231 \mathrm{rad}$} \\
\hline
\end{tabular}

Table 5: Geometric parameter identified values for DH SCARA first and second-order geometric model.

The residual errors after identification for points $\mathbf{M}_{\mathbf{1}}$ and $\mathbf{M}_{\mathbf{2}}$ are given in Figure 14. The maximum residual error value is obtained for a movement of joint 1 and is near $0.087 \mathrm{~mm}$. The mean residual error for a joint 1 movement is $0.041 \mathrm{~mm}$ and $0.010 \mathrm{~mm}$ for a joint 2 movement.
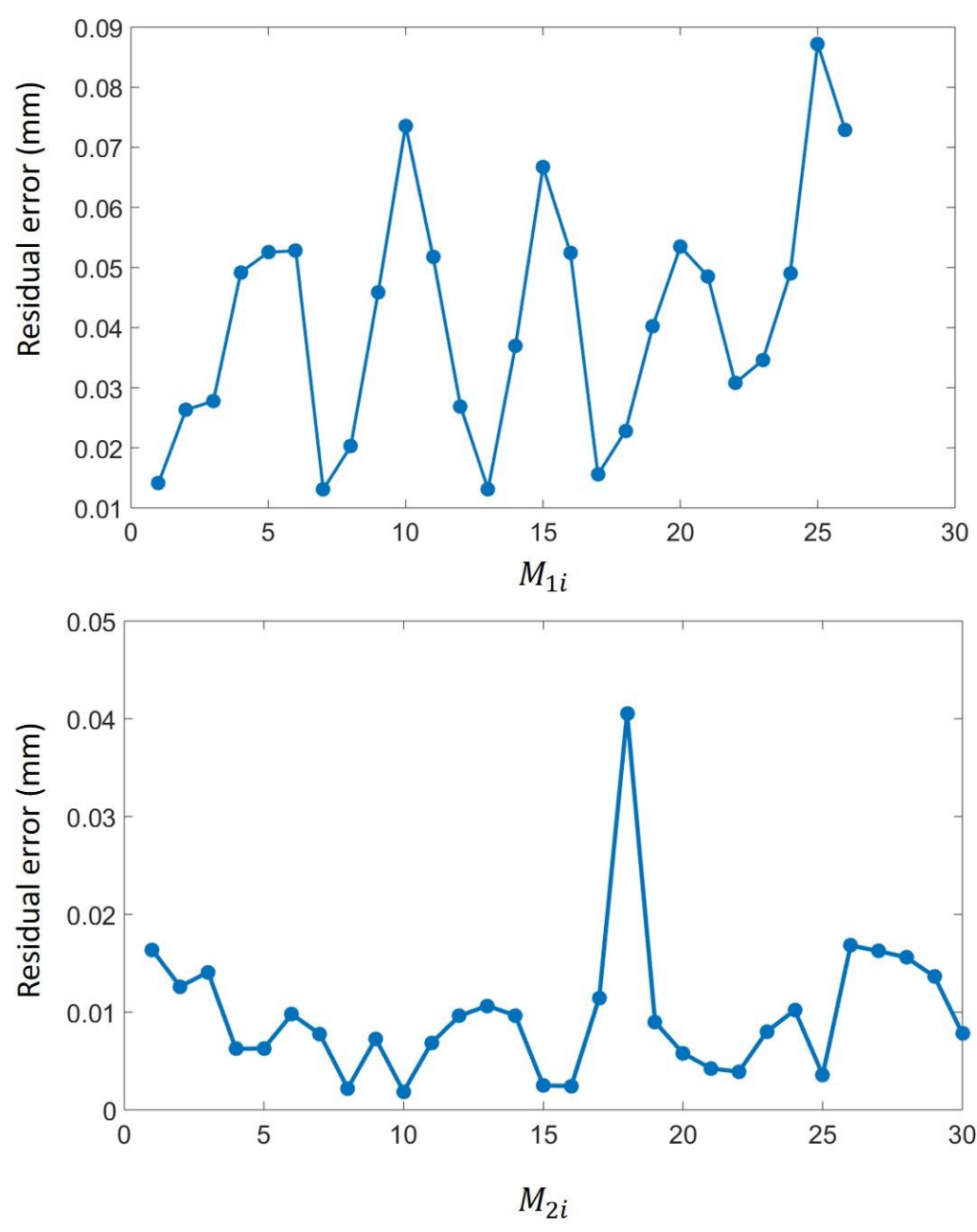

Figure 14: Residual errors for DH SCARA first and second-order geometric model. 
The same nine validation points are used to estimate the accuracy benefit brings by the vector geometric model (Figure 15). The errors between measured points position and simulated with identified DKM are less than $0.055 \mathrm{~mm}$. The defect is $0.009 \mathrm{~mm}$ higher compared to the proposed vector method.

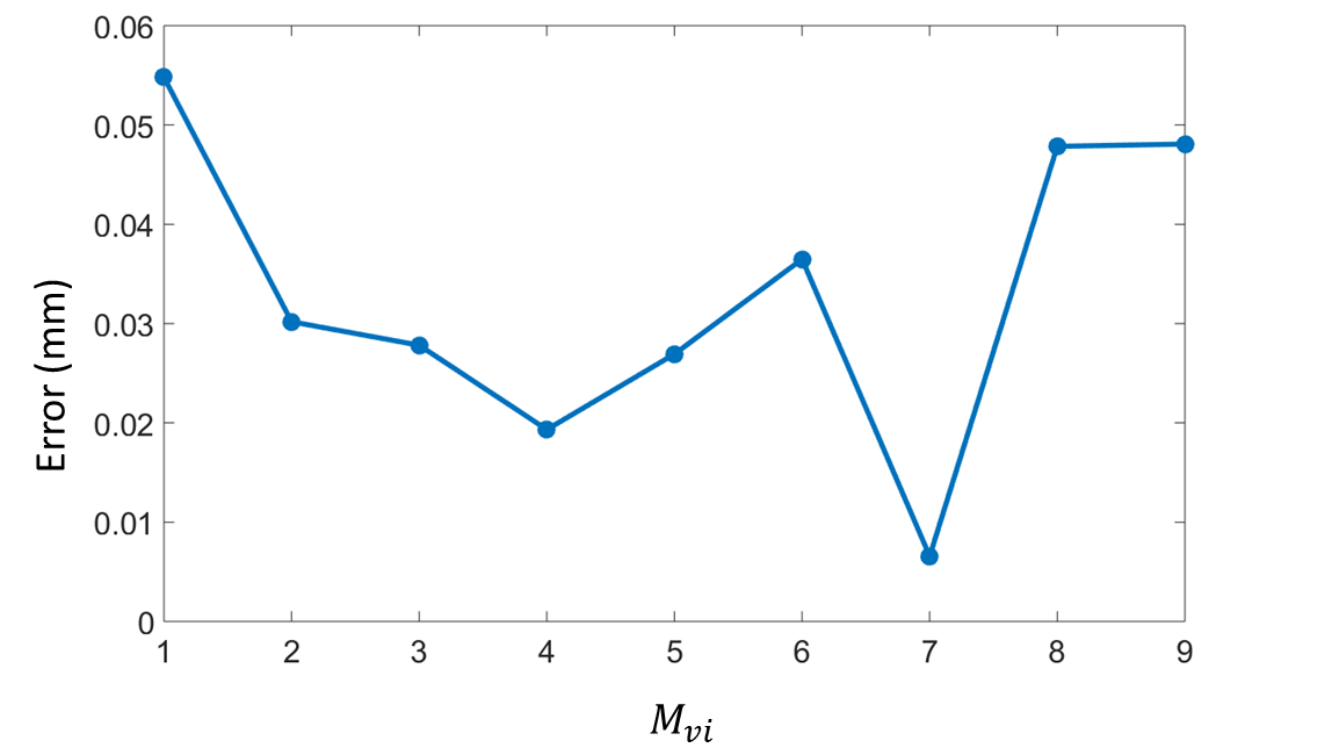

Figure 15: Error between measured position points and simulated with identified DH first and second-order DKM.

Indeed, in this case, a first step, before numerical optimization, should be realized to identify the position and orientation of the coordinate system $R_{1}$ regarding the laser tracker coordinate system. Thus, identification errors should be greater than those obtained with a CPA method due to this first step [22].

To finalize this study, and to illustrate the influence of this DKM parameters, we choose to neglect the term $I_{2}$ in our vector model. Indeed, in the DH first and second-order DKM, this parameter is not introduced. To see the influence of this term, we compare the measured position of the nine validation points with the simulated points with the vector geometric model with $I_{2}=0$ (Figure 16). 


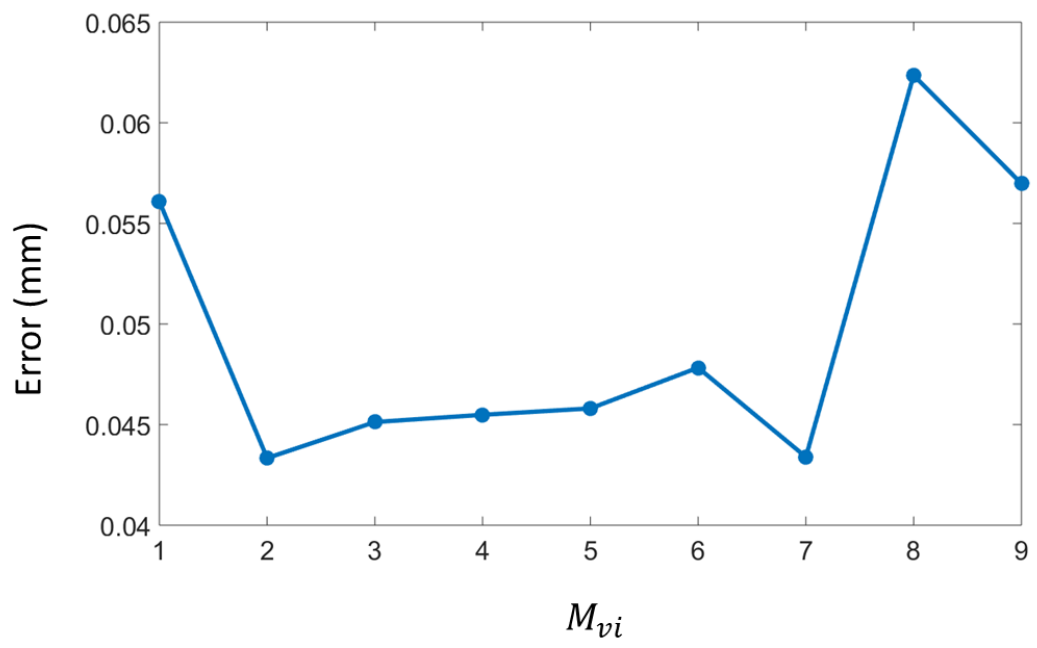

Figure 16: Error between measured position points and simulated with vector DKM with $I_{2}=$ 0 .

The errors between the position of the measured points and the studied DKM are less than $0.063 \mathrm{~mm}$. The defect is multiplied by two-thirds compared to the defect obtained with our vector method with $I_{2} \neq 0$ and is $0.008 \mathrm{~mm}$ higher compared to the $\mathrm{DH}$ first and second-order DKM. This last result validates the interest of the proposed vector model and shows the impact of taking into account all the orientation defect of joint axis.

\subsection{Summary}

To conclude on this section, the proposed vector modeling method improves the accuracy between the real geometric behavior and the simulated geometric behavior even if second-order defects are implemented in the DH geometric model.

Table 6 summarizes the obtained errors between each SCARA robot DKM simulation and the nine measured validation points. Note that the mean error of $0.027 \mathrm{~mm}$ obtained with our vector model is close to the measurement process accuracy.

\begin{tabular}{|c|c|c|}
\hline SCARA geometrical model & Max error $\mathbf{( m m )}$ & Mean error $(\mathbf{m m})$ \\
\hline Vector model & 0.046 & 0.027 \\
\hline DH first-order model & 0.122 & 0.098 \\
\hline DH first and second-order model & 0.055 & 0.033 \\
\hline
\end{tabular}

Table 6: Errors between the position of the measured points and the different test DKM

The other benefit of the proposed vector modeling is the consistency between the vector definition of each joint axis and the CPA identification process. This particularity decreases the influence of the identification numerical process on the inverse kinematic residual error.

\section{Conclusion}

In this article, a new geometric vector modeling method of a serial kinematic robot consistent with the CPA identification process is presented. This method is based on the definition of position and orientation of the robot joints invariants. The invariant of the rotational joint is a straight-line (rotational joint axis orientation and a point). Thus, four parameters are introduced to position a point and orient a vector which are linked to robot joints. The methodology to define these parameters is introduced. These parameters are identified from a CPA method. This identification ensures to directly identify a vector orientation and a point position with a geometrical point of view. 
The application of this method improves the final accuracy of the DKM regarding the DH convention. This method allows controlling the number of introduced geometric parameters even for adjacent parallel joints and also increase the accuracy obtained after the identification process.

These first results are promising regarding the impact of the implementation of this new geometric vector model on the obtained geometric accuracy of serial kinematic robot. Further works should now be conducted to apply this vector geometry modeling method to a closedloop robot.

\section{References}

[1] X. Yang, L. Wu, J. Li, K. Chen, A minimal kinematic model for serial robot calibration using POE formula, Robot. Comput. Integr. Manuf. 30 (2014) 326-334. https://doi.org/10.1016/j.rcim.2013.11.002.

[2] W. Khalil, E. Dombre, Modeling Identification and Control of Robots, ButterworthHeinemann, 2004. https://doi.org/10.1016/B978-1-903996-66-9.X5000-3.

[3] Y. Wu, A. Klimchik, S. Caro, B. Furet, A. Pashkevich, Geometric calibration of industrial robots using enhanced partial pose measurements and design of experiments, Robot. Comput. Integr. Manuf. 35 (2015) 151-168. https://doi.org/10.1016/j.rcim.2015.03.007.

[4] A. Omodei, G. Legnani, R. Adamini, Three methodologies for the calibration of industrial manipulators: Experimental results on a SCARA robot, J. Robot. Syst. 17 (2000) 291-307. https://doi.org/10.1002/(SICI)1097-4563(200006)17:6<291::AIDROB1>3.0.CO;2-U.

[5] M. Barati, A.R. Khoogar, M. Nasirian, Estimation and Calibration of Robot Link Parameters with Intelligent Techniques, Iran. J. Electr. Electron. Eng. 7 (2011) 225-234.

[6] Y. Cho, H.M. Do, J. Cheong, Screw based kinematic calibration method for robot manipulators with joint compliance using circular point analysis, Robot. Comput. Integr. Manuf. 60 (2019) 63-76. https://doi.org/10.1016/j.rcim.2018.08.001.

[7] J. Denavit, R.S. Hartenberg, A kinematic notation for lower pair mechanism based on matrices, Trans. ASME, J. Appl. Mech. 22 (1955) 215-221.

[8] G. Gogu, P. Coiffet, A. Barraco, Représentation des déplacements des robots, Hermes Science Publications, 1997.

[9] H. Lipkin, A note on Denavit-Hartenberg notation in robotics, in: Proc. ASME Int. Des. Eng. Tech. Conf. Comput. Inf. Eng. Conf. - DETC2005, 2005: pp. 921-926. https://doi.org/10.1115/detc2005-85460.

[10] L.J. Everett, M. Driels, B.W. Mooring, kinematic modelling for robot calibration, in: IEEE Int. Conf. Robot. Autom., 1987: pp. 183-189.

[11] W.K. Veitschegger, C.-H. Wu, Robot Accuracy Analysis Based on Kinematics, IEEE J. Robot. Autom. 3 (1986) 171-179. https://doi.org/10.1109/JRA.1986.1087054.

[12] K. Schröer, S.L. Albright, M. Grethlein, Complete, minimal and model-continuous kinematic models for robot calibration, Robot. Comput. Integr. Manuf. 13 (1997) 73-85. https://doi.org/10.1016/S0736-5845(96)00025-7.

[13] H. Chanal, E. Duc, J.Y. Hascoët, P. Ray, Reduction of a parallel kinematics machine tool 
inverse kinematics model with regard to machining behaviour, Mech. Mach. Theory. 44 (2009) 1371-1385. https://doi.org/10.1016/j.mechmachtheory.2008.11.004.

[14] K.C. Gupta, Kinematic Analysis of Manipulators Using the Zero Reference Position Description, Int. J. Rob. Res. 5 (1986) 5-13.

[15] R. He, Y. Zhao, S. Yang, S. Yang, Kinematic-parameter identification for serial-robot calibration based on POE formula, IEEE Trans. Robot. 26 (2010) 411-423. https://doi.org/10.1109/TRO.2010.2047529.

[16] I.M. Chen, G. Yang, C.T. Tan, S.H. Yeo, Local POE model for robot kinematic calibration, Mech. Mach. Theory. 36 (2001) 1215-1239. https://doi.org/10.1016/S0094114X(01)00048-9.

[17] A. Müller, An overview of formulae for the higher-order kinematics of lower-pair chains with applications in robotics and mechanism theory, Mech. Mach. Theory. 142 (2019). https://doi.org/10.1016/j.mechmachtheory.2019.103594.

[18] A. Clément, C. Valade, A. Rivière, The TTRS: 13 oriented constraints for dimensioning, tolerancing and inspection, Adv. Math. Tools Metrol. III. (1997) 24-42.

[19] W. Khalil, J.-F. Kleinfinger, A new geometric notation for open and close-loop robots, in: Conf. Int. Robot. Autom., USA, 1986: pp. 1174-1179.

[20] B. Siciliano, O. Khatib, Handbook of Robotics, Springer, 2008.

[21] B.W. Mooring, Z.S. Roth, M.R. Driels, Fundamentals of Manipulator Calibration, Wiley-Interscience Publication, 1991.

[22] H. Chanal, F. Paccot, E. Duc, Sensitivity analysis of an overconstrained parallel structure machine tool, the Tripteor X7, Applied Mechanics and Materials, 2012. https://doi.org/10.4028/www.scientific.net/AMM.162.394.

Appendix A: measured points in laser tracker coordinate system for SCARA robot geometric identification

\begin{tabular}{|c|c|c|c|c|c|c|c|c|c|c|c|}
\hline$M_{1 i}$ & $X$ & $Y$ & $Z$ & $q_{1}$ & $q_{2}$ & $M_{2 i}$ & $X$ & $Y$ & $Z$ & $q_{1}$ & $q_{2}$ \\
\hline 1 & -746.893 & 1682.145 & -410.693 & $-15^{\circ}$ & $-30^{\circ}$ & & -407.143 & 1673.864 & -413.804 & $50^{\circ}$ & $-100^{\circ}$ \\
\hline 2 & -713.586 & 1644.155 & -411.121 & $-10^{\circ}$ & $-30^{\circ}$ & & -398.993 & 1651.404 & -413.958 & $50^{\circ}$ & -995 \\
\hline 3 & -713.584 & 1644.154 & -411.124 & $-10^{\circ}$ & $-30^{\circ}$ & & -388.905 & 1629.712 & -414.130 & $50^{\circ}$ & $-9^{\circ} 0$ \\
\hline 4 & -677.066 & 1609.223 & -411.584 & $-5^{\circ}$ & $-30^{\circ}$ & & -376.952 & 1608.986 & -414.313 & $50^{\circ}$ & $-85^{\circ}$ \\
\hline 5 & -637.671 & 1577.610 & -412.056 & $0^{\circ}$ & $-30^{\circ}$ & & -363.247 & 1589.378 & -414.507 & $50^{\circ}$ & $-80^{\circ}$ \\
\hline 6 & -637.667 & 1577.614 & -412.050 & $0^{\circ}$ & $-30^{\circ}$ & & -347.870 & 1571.041 & -414.720 & $50^{\circ}$ & $-75^{\circ}$ \\
\hline 7 & -595.708 & 1549.577 & -412.534 & $5^{\circ}$ & $-30^{\circ}$ & & -347.872 & 1571.043 & -414.717 & $50^{\circ}$ & $-75^{\circ}$ \\
\hline 8 & -551.417 & 1525.281 & -413.035 & $10^{\circ}$ & $-30^{\circ}$ & & -330.978 & 1554.116 & -414.936 & $50^{\circ}$ & $-70^{\circ}$ \\
\hline 9 & -505.156 & 1504.940 & -413.533 & $15^{\circ}$ & $-30^{\circ}$ & & 666 & 1538.733 & -415.162 & $50^{\circ}$ & $-65^{\circ}$ \\
\hline 10 & -457.302 & 1488.706 & -414.047 & $20^{\circ}$ & $-30^{\circ}$ & & -293.079 & 1524.993 & -415.391 & $50^{\circ}$ & $-60^{\circ}$ \\
\hline 11 & -457.322 & 1488.708 & -414.027 & $20^{\circ}$ & $-30^{\circ}$ & & -272.367 & 1513.017 & -415.628 & $50^{\circ}$ & $-55^{\circ}$ \\
\hline 12 & -408.285 & 1476.713 & -414.520 & $25^{\circ}$ & $-30^{\circ}$ & & -250.696 & 1502.898 & -415.870 & $50^{\circ}$ & $-50^{\circ}$ \\
\hline 13 & -358.383 & 1469.036 & -415.013 & $30^{\circ}$ & $-30^{\circ}$ & & -250.709 & 1502.900 & -415.861 & $50^{\circ}$ & $-50^{\circ}$ \\
\hline 14 & -307.954 & 1465.736 & -415.491 & $35^{\circ}$ & $-30^{\circ}$ & & -228.221 & 1494.696 & -416.103 & $50^{\circ}$ & $-45^{\circ}$ \\
\hline 15 & -257.432 & 1466.844 & -415.971 & $40^{\circ}$ & $-30^{\circ}$ & & -205.132 & 1488.490 & -416.343 & $50^{\circ}$ & $-40^{\circ}$ \\
\hline 16 & -207.235 & 1472.356 & -416.404 & $45^{\circ}$ & $-30^{\circ}$ & & -181.579 & 1484.319 & -416.578 & $50^{\circ}$ & $-35^{\circ}$ \\
\hline 17 & -157.735 & 1482.214 & -416.817 & $50^{\circ}$ & $-30^{\circ}$ & & -133.817 & 1482.200 & -417.037 & $50^{\circ}$ & $-25^{\circ}$ \\
\hline
\end{tabular}




\begin{tabular}{|r|r|r|r|l|l|l|l|l|l|l|}
\hline $\mathbf{1 8}$ & -109.238 & 1496.356 & -417.214 & $55^{\circ}$ & $-30^{\circ}$ & -109.959 & 1484.267 & -417.263 & $50^{\circ}$ & $-20^{\circ}$ \\
\hline $\mathbf{1 9}$ & -62.150 & 1514.673 & -417.590 & $60^{\circ}$ & $-30^{\circ}$ & -109.989 & 1484.269 & -417.251 & $50^{\circ}$ & $-20^{\circ}$ \\
\hline $\mathbf{2 0}$ & -16.843 & 1537.025 & -417.93 & $65^{\circ}$ & $-30^{\circ}$ & -86.444 & 1488.406 & -417.459 & $50^{\circ}$ & $-15^{\circ}$ \\
\hline $\mathbf{2 1}$ & -16.850 & 1537.026 & -417.937 & $65^{\circ}$ & $-30^{\circ}$ & -63.332 & 1494.581 & -417.655 & $50^{\circ}$ & $-10^{\circ}$ \\
\hline $\mathbf{2 2}$ & 26.304 & 1563.223 & -418.245 & $70^{\circ}$ & $-30^{\circ}$ & -40.848 & 1502.747 & -417.838 & $50^{\circ}$ & $-5^{\circ}$ \\
\hline $\mathbf{2 3}$ & 67.023 & 1593.085 & -418.523 & $75^{\circ}$ & $-30^{\circ}$ & -19.165 & 1512.836 & -418.008 & $50^{\circ}$ & $0^{\circ}$ \\
\hline $\mathbf{2 4}$ & 105.006 & 1626.403 & -418.767 & $80^{\circ}$ & $-30^{\circ}$ & 1.575 & 1524.788 & -418.162 & $50^{\circ}$ & $5^{\circ}$ \\
\hline $\mathbf{2 5}$ & 139.966 & 1662.905 & -418.981 & $85^{\circ}$ & $-30^{\circ}$ & 1.563 & 1524.790 & -418.164 & $50^{\circ}$ & $5^{\circ}$ \\
\hline $\mathbf{2 6}$ & 171.539 & 1702.305 & -419.133 & $90^{\circ}$ & $-30^{\circ}$ & 21.153 & 1538.489 & -418.302 & $50^{\circ}$ & $10^{\circ}$ \\
\hline $\mathbf{2 7}$ & & & & & & 39.488 & 1553.856 & -418.421 & $50^{\circ}$ & $15^{\circ}$ \\
\hline $\mathbf{2 8}$ & & & & & & 56.416 & 1570.765 & -418.523 & $50^{\circ}$ & $20^{\circ}$ \\
\hline $\mathbf{2 9}$ & & & & & & 71.808 & 1589.067 & -418.606 & $50^{\circ}$ & $25^{\circ}$ \\
\hline $\mathbf{3 0}$ & & & & & & 85.547 & 1608.663 & -418.667 & $50^{\circ}$ & $30^{\circ}$ \\
\hline
\end{tabular}

Annex B: Measured points for validation of the identification process

\begin{tabular}{|r|r|r|r|r|r|}
\hline $\boldsymbol{M}_{\boldsymbol{i}}$ & $\boldsymbol{X}$ & $\boldsymbol{Y}$ & $\boldsymbol{Z}$ & \multicolumn{1}{c|}{$\boldsymbol{q}_{\mathbf{1}}$} & \multicolumn{1}{c|}{$\boldsymbol{q}_{\mathbf{2}}$} \\
\hline $\mathbf{1}$ & -465.303 & 1493.982 & -413.932 & $20^{\circ}$ & $-32^{\circ}$ \\
\hline $\mathbf{2}$ & -571.505 & 1545.766 & -412.775 & $10^{\circ}$ & $-36^{\circ}$ \\
\hline $\mathbf{3}$ & -513.505 & 1496.169 & -413.491 & $10^{\circ}$ & $-20^{\circ}$ \\
\hline $\mathbf{4}$ & -567.966 & 1523.226 & -412.897 & $5^{\circ}$ & $-22^{\circ}$ \\
\hline $\mathbf{5}$ & -520.183 & 1491.571 & -413.451 & $5^{\circ}$ & $-10^{\circ}$ \\
\hline $\mathbf{6}$ & -394.761 & 1453.646 & -414.745 & $13^{\circ}$ & $0^{\circ}$ \\
\hline $\mathbf{7}$ & -12.934 & 1526.406 & -418.046 & $42^{\circ}$ & $20^{\circ}$ \\
\hline $\mathbf{8}$ & 135.229 & 1638.539 & -419.056 & $61^{\circ}$ & $18^{\circ}$ \\
\hline $\mathbf{9}$ & 131.630 & 1624.232 & -419.053 & $68^{\circ}$ & $0^{\circ}$ \\
\hline
\end{tabular}

